\title{
Overcoming Chemoresistance via Extracellular Vesicle Inhibition
}

\section{OPEN ACCESS}

Edited by:

Miroslaw Kornek,

Universität Bonn, Germany

Reviewed by:

Carolina Panis,

Universidade Estadual do Oeste do

Paraná, Brazil

Jose J. G. Marin

University of Salamanca, Spain

${ }^{*}$ Correspondence:

Wai-Leng Lee

lee.wai.leng@monash.edu

Nurolaini Kifl

nurolaini.kifli@ubd.edu.bn

Specialty section:

This article was submitted to

Molecular Diagnostics

and Therapeutics,

a section of the journal

Frontiers in Molecular Biosciences

Received: 16 November 2020

Accepted: 22 February 2021

Published: 24 March 2021

Citation:

Hayatudin R, Fong Z, Ming LC,

Goh B-H, Lee W-L and Kifli N (2021)

Overcoming Chemoresistance via

Extracellular Vesicle Inhibition.

Front. Mol. Biosci. 8:629874.

doi: 10.3389/fmolb.2021.629874

\author{
Raeesah Hayatudin', Zhijack Fong ${ }^{1}$, Long Chiau Ming², Bey-Hing Goh ${ }^{3,4}$, \\ Wai-Leng Lee ${ }^{1 *}$ and Nurolaini Kifli ${ }^{2 *}$ \\ ${ }^{1}$ School of Science, Monash University Malaysia, Subang Jaya, Malaysia, ${ }^{2}$ PAP Rashidah Sa'adatul Bolkiah Institute \\ of Health Sciences, Universiti Brunei Darussalam, Gadong, Brunei, ${ }^{3}$ College of Pharmaceutical Sciences, Zhejiang \\ University, Hangzhou, China, ${ }^{4}$ Biofunctional Molecule Exploratory (BMEX) Research Group, School of Pharmacy, Monash \\ University Malaysia, Subang Jaya, Malaysia
}

With the ever-growing number of cancer deaths worldwide, researchers have been working hard to identify the key reasons behind the failure of cancer therapies so the efficacy of those therapies may be improved. Based on extensive research activities and observations done by researchers, chemoresistance has been identified as a major contributor to the drastic number of deaths among cancer patients. Several factors have been linked to formation of chemoresistance, such as chemotherapy drug efflux, immunosuppression, and epithelial-mesenchymal transition (EMT). Lately, increasing evidence has shed light on the role of extracellular vesicles (EVs) in the regulation of chemoresistance. However, there is limited research into the possibility that inhibiting EV release or uptake in cancer cells may curb chemoresistance, allowing chemotherapy drugs to target cancer cells without restriction. Prominent inhibitors of EV uptake and release in cancer cells have been compiled and contrasted in this review. This is in the hope of sparking greater interest in the field of EV-mediated chemoresistance, as well as to provide an overview of the field for fundamental and clinical research communities, particularly in the field of cancer resistance research. In-depth studies of EV-mediated chemoresistance and EV inhibitors in cancer cells would spur significant improvement in cancer treatments which are currently available.

\section{Keywords: chemoresistance, extracellular vesicle, cancer, exosomes, cell death}

\section{INTRODUCTION}

Chemoresistance, a state of cancer cells wherein chemotherapy drugs are ineffective against cancer progression, is a major challenge in cancer treatment. It accounts for more than $90 \%$ of deaths in patients with metastatic tumors (Wang et al., 2019). Chemoresistance in tumors may be either intrinsic or acquired. Acquired chemoresistance may cause cancer cells to develop insensitivity not only to the particular chemotherapy drug being used, but also to other types of chemotherapy drugs with different modes of action (Wang et al., 2019). Many chemotherapy drugs function by targeting DNA replication, which prevents the growth of cancer cells and induces apoptosis - for example, cisplatin intercalates with double-stranded DNA whereas 5-fluorouracil inhibits DNA nucleotide synthesis (Chorowala et al., 2012). However, chemoresistant cells respond to these drugs by developing mechanisms which promote their proliferation and inhibit apoptotic processes. 
These mechanisms have recently been proven with the involvement of extracellular vesicles (EVs) (Namee and O'driscoll, 2018; Santos et al., 2018).

This review will compile major drug inhibitors targeting specific mechanisms in the release and uptake of both exosomes and microvesicles (MVs) from cancer cells. Existing literature regarding the effectiveness of these inhibitors, as well as the possible difficulties in using them, will be analyzed. This is with the aim of emphasizing the potential of using EV inhibitors to target chemoresistance in cancer therapies.

\section{Subtypes of EVs}

Extracellular vesicles are small, lipid bilayer-enclosed particles secreted from all types of cells for intercellular communication and the removal of cellular wastes; in cancer cells, they mediate chemoresistance (Fan et al., 2018) as well as cancer progression (Xu et al., 2018). EVs exist in a diverse range of sizes even within subclassifications. Their constituents (a mix of nucleic acids, proteins and lipids) may vary depending on the subtype of $\mathrm{EV}$, the function of the parent cell (Zaborowski et al., 2015), the stage of cell growth or pathogenesis the parent cell is undergoing (Becker et al., 2016), and even whether EVs are secreted from the apical or basolateral surfaces of polarized cells (Tauro et al., 2013; Chen et al., 2016). The two most well-categorized EV subtypes concerning chemoresistance are exosomes and MVs. Exosomes are typically defined as EVs falling within the size range of 30 to $100 \mathrm{~nm}$, resulting directly from endosomal multivesicular bodies (MVBs); whereas MVs are classified as being within 100 to $1000 \mathrm{~nm}$ and outwardly budding from cell membranes (Doyle and Wang, 2019; Ciardiello et al., 2020). Nevertheless, EVs in the size range of exosomes have been found to occasionally derive from outward budding rather than endosomal MVBs, though not yet in cancer cell lines (Lenassi et al., 2010; Abels and Breakefield, 2016).

\section{Biogenesis and Release of EVs}

Exosomes are formed via the fusion of MVBs with the plasma membrane; MVBs result from the inward budding of endosomal membranes (Huotari and Helenius, 2011). MVBs are assembled via endosomal sorting complexes required for transport (ESCRT)-independent and ESCRT-dependent pathways (Huotari and Helenius, 2011). In the ESCRTindependent pathway, sphingomyelinase enzymes (SMases) convert sphingomyelin (present in endosomal membranes within lipid rafts) to ceramide (Catalano and O'driscoll, 2020). The ceramides which associate to form microdomains which drive the formation of MVBs from intra-luminal vesicles (ILVs) (Huotari and Helenius, 2011). Meanwhile, the ESCRT-dependent pathway requires ESCRT protein complexes (specifically, ESCRT0, ESCRT-I, ESCRT-II, and ESCRT-III) with associated proteins (such as VPS4, VTA1, and ALIX) which aid in the generation of ILVs (Huotari and Helenius, 2011). ESCRT-0 gathers ubiquitinated proteins and recruits ESCRT-I and ESCRT-II, which cooperate to contort the endosomal membrane and sort the constituents of the forming ILV (Hanson and Cashikar, 2012; Taylor and Bebawy, 2019). ESCRT-III component proteins are gathered by ESCRT-II or ALIX, and polymerization of those components causes neck constriction and cleavage to form ILVs (Catalano and O'driscoll, 2020). Vsp4, an ATPase, disassembles the ESCRT and allows them to be recycled (Babst, 2011). Farnesyltransferase enzymes are necessary for the activation of Ras proteins, which, together with its downstream effectors (including Raf and extracellular signal-regulated kinases, ERK), have been implicated in exosome biogenesis (Sexton et al., 2019). The MVBs formed which do not fuse with lysosomes are transported toward the cell membrane with the aid of cytoskeletal actin and microtubules; this process is regulated by several proteins such as the Rab proteins (Bobrie et al., 2012), and cholesterol impacts it as well (Pfrieger and Vitale, 2018). It should be noted that the ESCRT-independent and ESCRTdependent pathways might actually coordinate together in the biogenesis of exosomes rather than function distinctly (Babst, 2011; Hessvik and Llorente, 2018). Genetic editing to knock out the components of ESCRT-independent and ESCRT-dependent mechanisms would help to clarify whether those components are truly necessary for exosome biogenesis, and may reveal the extent to which the mechanisms cooperate (Maas et al., 2017).

Microvesicles form via budding directly from the plasma membrane. The transport of molecular constituents of MVs toward the plasma membrane as well as the rearrangement of membrane lipids and actin cytoskeletal components are integral to this process (Hessvik and Llorente, 2018). Several unique mechanisms involving lipids relate to MV release, including the externalization of phosphatidylserine (PS) at specific locations of the cell membrane where microvesiculation occur (Tricarico et al., 2017), as well as acid sphingomyelinase (aSMase)-catalyzed conversion of sphingomyelin to ceramide, which induces the curving of the plasma membrane and subsequent MV release (Muralidharan-Chari et al., 2010; Awojoodu et al., 2014; Hoehn et al., 2017; Menck et al., 2017). Cholesterol is involved in MV release in addition to intracellular transport of MVBs, although its precise role is unknown (Pfrieger and Vitale, 2018). Cytoskeleton-interacting components and regulators of those components also participate in the biogenesis of MVs. These include the Rho family of GTPases, which takes part in many cell signaling processes such as cytoskeleton reorganization (Catalano and O'driscoll, 2020), most prominently among them, RhoA, which acts on Rho-associated protein kinases (ROCK) and ERK (Li et al., 2012; Sedgwick et al., 2015; Hoehn et al., 2017). ROCK acts on the cytoskeleton to influence the cellular shape and movement (Hartmann et al., 2015). RhoA acts through the RhoAcofilin pathway to regulate the rearrangement of cytoskeletal actin in microvesiculation (Wilson et al., 2013). RhoA along with ADP-ribosylation factors 6 and 1 (ARF6 and ARF1) additionally increase myosin contractility via phosphorylation of myosin light chains, hence encouraging fission and the release of MVs (Schlienger et al., 2014). In addition, ESCRT proteins directly participate in $\mathrm{MV}$ biogenesis by regulating the cleavage of the plasma membrane to release MVs (Nabhan et al., 2012; Hurley, 2015; Wang and Lu, 2017; Arii et al., 2018; Taylor and Bebawy, 2019). In this way, MV biogenesis parallels the formation of ILVs, nonetheless, while the involvement of ESCRT in ILV formation is clear, more investigation is required regarding the role of ESCRT in MV budding. Moreover, this suggests that 
the distinction between the pathways of exosome biogenesis versus those of microvesiculation may not always be so clear-cut. More research is needed in order to elucidate the mechanisms of microvesiculation.

\section{Uptake of EVs}

Past research (Tian et al., 2014; Costa Verdera et al., 2017) has shown that EVs (including exosomes and MVs) are endocytosed by cancer cells through two main routes of pinocytosis, which can be broadly classified as clathrin-dependent endocytosis (CDE) and clathrin-independent endocytosis (CIE). In CDE, major coat proteins and endocytic accessory proteins assemble to form clathrin-coated pits and the GTPase dynamin assists in the scission of endocytic vesicles (Mettlen et al., 2018). In comparison, CIE encompasses a number of pathways including macropinocytosis as well as dynamin-independent processes such as CDC42- and ARF6-dependent endocytosis, and dynamin-dependent processes such as caveolae- and RhoA-dependent endocytosis (Dutta and Donaldson, 2012; Iversen et al., 2012; Costa Verdera et al., 2017). Many of the aforementioned variations of CIE are cholesteroldependent (Dutta and Donaldson, 2012). Several modes of macropinocytosis exist which may be dynamin-dependent and dynamin-independent (Hetzenecker et al., 2016; Sandvig et al., 2018), although further research is needed to clarify these mechanisms. Investigations into whether CDE or CIE are more significant in the uptake of EVs by cancer cells have yielded conflicting results, depending on the types of cell lines used. For example, in mesenchymal cells taking up PC12 (rat pheochromocytoma)-derived exosomes, CDE, and macropinocytosis (but not any other pathways of CIE) were implicated in uptake processes (Tian et al., 2014). However, for Jeko-1 and Mino (mantle cell lymphoma) cells (Hazan-Halevy et al., 2015), as well as A431 (human epidermoid carcinoma) and HeLa cells (Costa Verdera et al., 2017), EVs were described broadly to be internalized via CIE, but not CDE. EV uptake mechanisms in cancer cells may depend on cell types which produce and accept EVs.

\section{Regulation of Chemoresistance by EVs}

There are various mechanisms by which EVs confer chemoresistance from chemoresistant cancer cells to chemosensitive cells. These mechanisms have been summarized in Figure 1. As per Figure 1, EVs may modulate chemoresistance by transferring vesicular content, especially microRNAs (miRNAs), which activate anti-apoptotic signaling and DNA damage repair (DDR) (miR-21), and by enhancing other processes such as chemotherapy drug efflux (miR-1246), immunosuppression (miR-21), alteration of cytosolic $\mathrm{pH}$ and epithelial-mesenchymal transition (EMT) (miR-21) (Cereghetti and Lee, 2014; Yousafzai et al., 2018; Maacha et al., 2019; O'neill et al., 2019).

MicroRNAs, small single-stranded non-coding RNAs about 19-25 nucleotides long, contribute to the development of chemoresistance by influencing genes relating to drug resistance, cell survival and proliferation, cell cycle, apoptosis, stress tolerance and immunity (Si et al., 2019). MiRNAs regulate various genes via inhibiting mRNA translation. In EV-mediated chemoresistance, many studies suggested miRNA play important roles in the process. For instance, paclitaxel resistant ovarian cancer cells were reported to secrete EVs with abundance of miR-1246. Transfer of this miRNA promotes drug resistance phenotype in recipient cells by inhibiting the expression of Cav1 and upregulating $\mathrm{ABCB} 1$ expression to facilitate paclitaxel efflux (Kanlikilicer et al., 2018). Gemcitabine is a DNA chelator that is activated by deoxycytidine kinase. Several miRNAs were found to regulate the transferring of gemcitabine resistance. miR-365 in EVs secreted by tumor associated macrophages was observed to induce resistance of pancreatic ductal adenocarcinoma cells in gemcitabine treatment. This miRNA increased the concentration of triphosphate nucleotides (NTPs) in the recipient cells to allow more NTPs competing with activated gemcitabine, which effectively decreased the efficacy of gemcitabine (Binenbaum et al., 2018). On the other hand, increased miR-106 was found in EVs secreted by cancer associated fibroblasts when exposed to gemcitabine. Uptake of miR-106 enriched EVs contributes to the resistance of pancreatic cancer cells (AsPC1) against gemcitabine treatment. miR-106 conferred resistance toward gemcitabine by binding onto its target gene, TP53INP1, suppressing the expression of the protein (Fang et al., 2019).

Extracellular vesicles may additionally contribute to producing a chemoresistive tumor microenvironment (Maacha et al., 2019). miRNA in EVs could alter chemotherapy response and promote tumorigenesis by mediating the intracellular communication between the tumor and healthy cells. For examples, the tumor-derived EVs in patients with hepatocellular carcinoma (HEPG2) was found to contain miRNA-124730, a miRNA facilitates metastatic invasion of the tumor. It promotes the differentiation of normal lung fibroblasts into tumor promoting cancer-associated cells. The cancer-associated fibroblasts further enhance expression of pro-inflammatory cytokines such as interleukin- 6 and interleukin- 8 in cancer cells that led to their resistance in sorafenib treatment (Fang et al., 2018). Together these findings implicate inhibition of EVs may solve the problem of insensitivity of cancer cells in response to clinical drugs like gemcitabine and sorafenib.

\section{Inhibition of EVs}

In investigations into EV-mediated chemoresistance, treatment of cancer cell lines with EV-inhibiting drugs may serve two functions: to confirm that EVs contribute to chemoresistance, or to test whether EV inhibition is a suitable strategy to complement treatment with chemotherapy drugs. Although clear links have been established regarding the contribution of EVs to chemoresistance, scientific understanding of the precise mechanisms associated remain primitive and requires more investigation. EV inhibitors which bind to certain proteins or deregulate genes implicated in inducing chemoresistance may be used to clarify the roles those proteins or genes play in chemoresistance. An EV-related strategy against chemoresistance which holds promise includes inhibiting the biogenesis, release or uptake of EVs by cancer cells. Another approach of extracting tumor-derived EVs out from the tumor microenvironment using specific markers for those EVs has also been proposed 


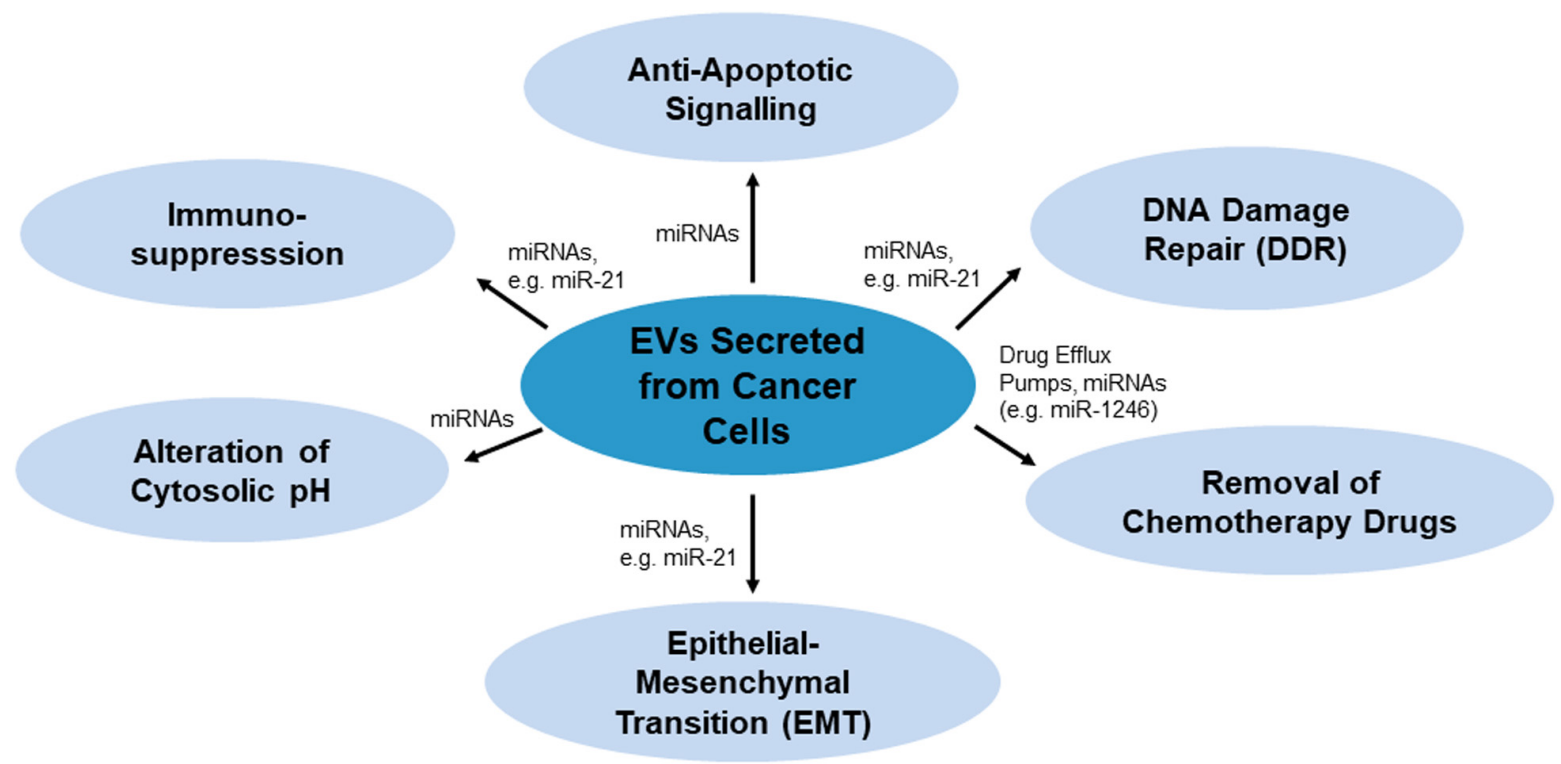

FIGURE 1 | Mechanisms of EVs-mediated chemoresistance miRNAs or proteins such as drug efflux pumps as the key players carrying drug resistance messages.

(Maacha et al., 2019), but this method has not been investigated for its efficacy using cancer cell lines at this time. While the inhibition of EV biogenesis, release or uptake may involve unique challenges due to the lack of specificity of some inhibitor drugs, it has proven efficient in inducing chemosensitivity to previously chemoresistant cancer cells. For instance, the kinase inhibitor U0126 sensitized chemoresistant Suit-2 (human pancreatic adenocarcinoma) cells to the chemotherapy drug gemcitabine, although it did not have as strong an effect on another chemoresistant pancreatic cancer cell line (Muralidharan-Chari et al., 2016). In the future, EV inhibition may prove to be an effective clinical strategy to counteract chemoresistance; for this purpose, the targeted pathways of EV production and uptake of EV-inhibiting drugs must be further understood.

To encourage further investigation, 33 inhibitors have been assessed according to the pathways of EV secretion or uptake which they primarily target. These pathways, as well as their corresponding inhibitors, have been summarized in Table 1, according to current knowledge about the activities of the inhibitors. Nevertheless, EV inhibitors may target more than one pathway, either due to their unspecificity or because the subject of the inhibitor may participate in multiple pathways of EV release and uptake. The inhibitors may target exosomes or MVs, or both.

The 33 inhibitors discussed may be broadly categorized as inhibitors of lipid-related pathways, cytoskeletal organization, other miscellaneous pathways of EV release and endocytosis. These four classifications have been selected based on the specific mechanisms targeted by each inhibitor.

\section{Inhibitors of Lipid-Related Pathways}

Various lipid-related mechanisms are associated with the release of EVs from cancer cells. These mechanisms primarily involve the conversion of sphingomyelin to ceramide by sphingomyelinases, PS translocation by protein kinases, and cholesterol synthesis. Ceramides play roles in causing the curvature of membranes prior to the formation of MVBs or release of MVs; PS translocation is a necessary step for MVs to be released; and cholesterol influences the formation of MVBs and MVs (Figure 2). Thus, inhibition of any of these pathways may hold promise in reducing EV release. If so, this may disable cancer cells from transmitting chemoresistance to other cancer cells.

\section{Inhibitors Targeting Sphingomyelinases}

Such inhibitors include imipramine, GW4869, cambinol, and spiroepoxide as depicted in Figure 2. GW4869, cambinol, and spiroepoxide selectively inhibit neutral sphingomyelinases (nSMase), more specifically neutral sphingomyelinase 2 (nSMase2); whereas imipramine targets aSMase. Each inhibitor may have a different specific mechanism, but they have all been proven to reduce exosome secretion, to varying levels of efficiency. For example, imipramine, an aSMase inhibitor, was found to block the release of exosomes and MVs from glial cells (Bianco et al., 2009). aSMases catalyze the hydrolysis of sphingomyelin to ceramide, which is vital to the release of exosomes and the formation of MVs. When MVs are shed, aSMases are translocated to the cell membrane; once internalized in cells, imipramine acts to proteolytically degrade aSMase. aSMase then detaches from the cell membrane (Catalano and O'driscoll, 2020). A study by Kosgodage et al. (2017) proved that $25 \mu \mathrm{M}$ imipramine lessened total EV release (both exosomes and MVs) by $77 \%$ in PC3 (prostate cancer) cells although the separate effects of imipramine on exosomes and on MVs was not tested (Kosgodage et al., 2017). Imipramine may also be used to inhibit macropinocytosis. 
TABLE 1 | List of EV inhibitors.

\begin{tabular}{|c|c|c|c|}
\hline Targets of inhibition & Reported inhibitors & Cell line or model used & References \\
\hline & Calpeptin & Cell line: PC3 & Jorfi et al., 2015 \\
\hline & Glyburide & Cell lines: PC3 and MCF-7 & Kosgodage et al., 2017 \\
\hline & NSC23766 & Sepsis model using mice & Wang et al., 2017 \\
\hline & บ0126 & Cell lines: Suit-2, MPanc-96 & Becker et al., 2016 \\
\hline \multirow[t]{12}{*}{ Formation of exosomes } & Cambinol $^{*}$ & Cell line: $\mathrm{NCl}-\mathrm{H} 460$ & Heltweg et al., 2006 \\
\hline & Cytochalasin D & Cell lines: HeLa, Panc 1, PC3, and A293 & Khan et al., 2011 \\
\hline & Dasatinib & Cell line: K562 & Mineo et al., 2012 \\
\hline & Dimethyl amiloride (DMA) & Cell lines: CT26, EL4, and H23 & Chalmin et al., 2010 \\
\hline & & $\begin{array}{l}\text { Model: three mouse tumor models using two } \\
\text { mouse cancer cell lines, EL4 and TS/A, and } \\
\text { one human cancer cell line, CT26 }\end{array}$ & \\
\hline & Ketotifen & Cell lines: HeLa, MCF-7 and BT549 & Khan et al., 2018 \\
\hline & Manumycin A & Cell lines: C4-2B, PC3, and 22Rv1 & Datta et al., 2017 \\
\hline & $\mathrm{M} \beta \mathrm{CD}$ & Cell lines: PC3 and MCF-7 & Kosgodage et al., 2017 \\
\hline & Simvastatin & Non-cancerous cell lines: Beas-2B and THP-1 & Kulshreshtha et al., 2019 \\
\hline & Spiroepoxide* & Cell line: PC3 & Phuyal et al., 2014 \\
\hline & Sulphisoxazole & Cell lines: MCF-7, MCF-10A, and MDA-MB-231 & Im et al., 2019 \\
\hline & Tipifarnib & $\begin{array}{l}\text { Cell line: modified C4-2B expressing exosomal } \\
\text { marker CD63 }\end{array}$ & Datta et al., 2018 \\
\hline \multirow[t]{2}{*}{$\begin{array}{l}\text { Formation of both MVs and } \\
\text { exosomes }\end{array}$} & Cannabidiol & $\begin{array}{l}\text { Cell lines: HEPG2, MDA-MB-231, and PC3 cell } \\
\text { lines }\end{array}$ & Kosgodage et al., 2018 \\
\hline & Chloramidine (Cl-amidine) & Cell lines: PC3 and MCF-7 & Kosgodage et al., 2017 \\
\hline $\begin{array}{l}\text { Clathrin-dependent } \\
\text { endocytosis (CDE) }\end{array}$ & $\mathrm{M} \beta \mathrm{CD}$ & Cell lines: PC3 and MCF-7 & Kosgodage et al., 2017 \\
\hline \multirow{4}{*}{$\begin{array}{l}\text { Clathrin-independent } \\
\text { endocytosis (CIE) }\end{array}$} & Genistein & Cell lines: A2780, CaOV3, ES2, and SK-OV-3 & Costa Verdera et al., 2017 \\
\hline & Heparin & Cell line: U87 & Christianson et al., 2013 \\
\hline & $\mathrm{M} \beta \mathrm{CD}$ & Cell lines: PC3 and MCF-7 & Kosgodage et al., 2017 \\
\hline & Simvastatin & Non-cancerous cell lines: Beas-2B and THP-1 & Kulshreshtha et al., 2019 \\
\hline \multirow{5}{*}{$\begin{array}{l}\text { Macropinocytosis (subset } \\
\text { of } \mathrm{CIE} \text { ) }\end{array}$} & Cytochalasin D & Cell lines: A431 and HeLa & Costa Verdera et al., 2017 \\
\hline & Dimethyl amiloride (DMA) & Cell lines: CT26, EL4 and H23 & Chalmin et al., 2010 \\
\hline & & $\begin{array}{l}\text { Model: three mouse tumor models using two } \\
\text { mouse cancer cell lines, EL4 and TS/A, and } \\
\text { one human cancer cell line, CT26 }\end{array}$ & \\
\hline & EIPA & Cell line: $4 \mathrm{~T} 1$ & Lin et al., 2018 \\
\hline & NSC23766 & Cell lines: MDA-MB-435 and MCF-10A & Hernandez et al., 2010 \\
\hline Both CDE and CIE & Chloramidine (Cl-amidine) & $\begin{array}{l}\text { Non-cancerous cell lines: HuH-7, Vero, COS-7, } \\
\text { ARPE-19, and D407 }\end{array}$ & Vercauteren et al., 2010 \\
\hline
\end{tabular}

*Inhibitors which have not yet been tested with cancer cells (categorization based on mechanism of inhibitor drug). 


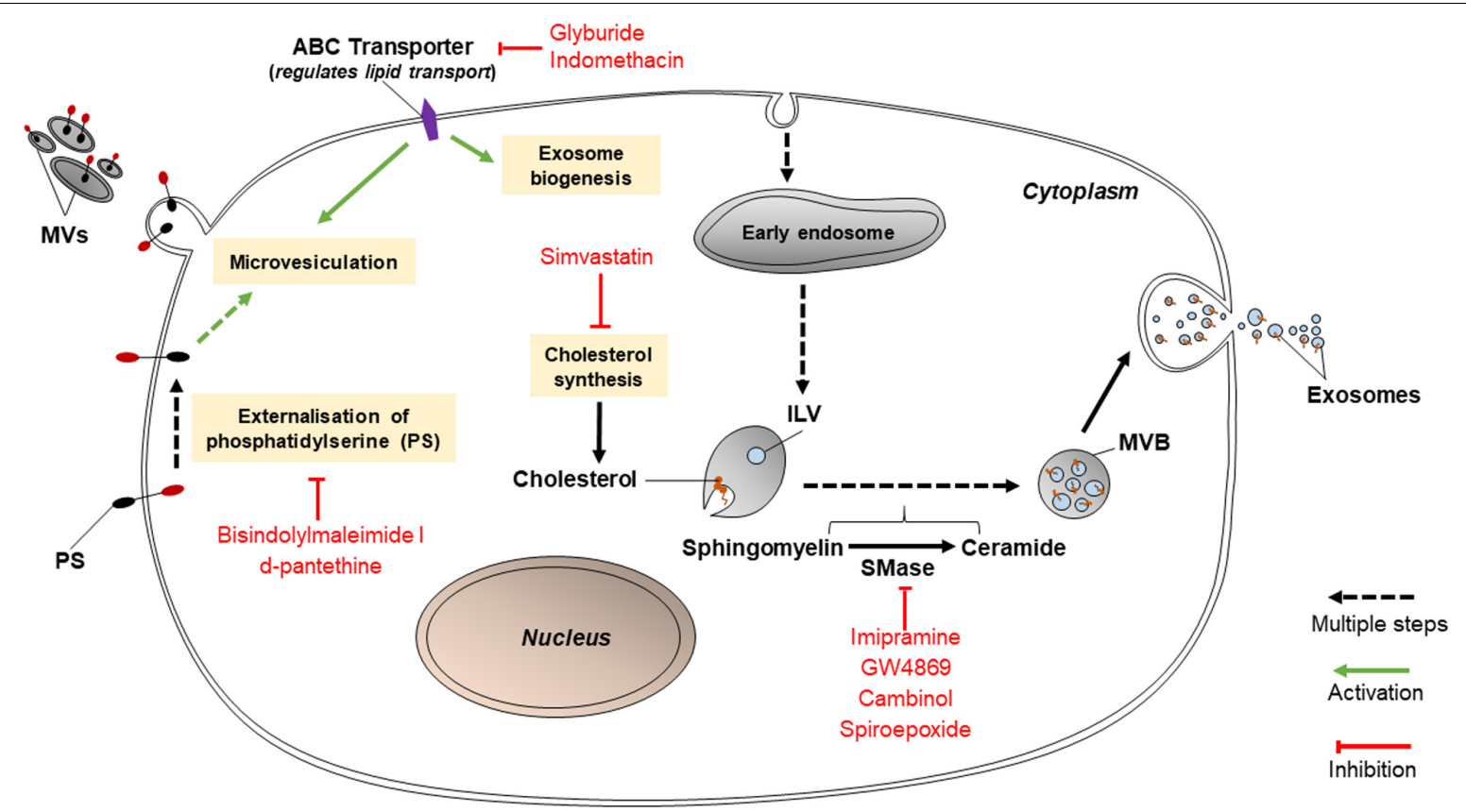

FIGURE 2 | Inhibitors of lipid-related pathways (bisindolylmaleimide-l, d-pantethine, glyburide, indomethacin, simvastatin, imipramine, GW4869, cambinol, and spiroepoxide) inhibit EV biogenesis.

nSMase2 inhibitors block ceramide-mediated exosome biogenesis. GW4869 is a frequently used inhibitor which targets nSMase 2 to prevent the ceramide-modulated inward budding of MVBs and the subsequent release of exosomes from MVBs (Catalano and O'driscoll, 2020). GW4869 was used to suppress chemoresistance in colorectal, pancreatic and ovarian cancer cells by reducing exosome secretion (Hu et al., 2015; Cao et al., 2017; Richards et al., 2017). Cambinol, a lipid molecule, binds to the active site of nSMase2 (Figuera-Losada et al., 2015). While its specific nSMase2 inhibitory effect on cancer-derived EVs has not been studied, on the basis of its additional inhibition of NAD-dependent deacetylase activity of cell survival enzymes, it has been found to have antitumorigenic effects on lung cancer cells from the cell line NCI-H460 as well as sensitize those cells to chemotherapy drugs such as DNA-damaging etoposide and tubulin-targeting paclitaxel (Heltweg et al., 2006). Spiroepoxide specifically and non-competitively inhibits nSMase2 (Phuyal et al., 2014) although its ability to diminish exosome secretion in cancer cells has not been documented. GW4869 and spiroepoxide have both been found to be non-toxic to cells (Phuyal et al., 2014). It must be noted that while nSMase 2 inhibitors have been proven to reduce exosome release in cancerous cell lines as well as some non-cancerous cell types such as HEK293 cells, a human kidney cell line (Kosaka et al., 2010), they do not affect exosome secretion at all in other cell types such as PC3, a prostate adenocarcinoma cell line (Phuyal et al., 2014). The efficiency of EV inhibitors varies between different cell lines and cell types.

\section{Inhibitors Targeting PS Translocation}

These include bisindolylmaleimide-I and d-pantethine. Bisindolylmaleimide-I, an inhibitor targeting the ATP-binding site of various protein kinase $\mathrm{C}$ isoforms, hinders the release of calcium and the externalization of PS, which are known steps in the mechanism driving the release of MVs (Kosgodage et al., 2017). Stratton et al. (2015) detailed that bisindolylmaleimide-I inhibited MV release in PC3 cells by $75 \%$ compared to the control, despite intracellular calcium concentrations being increased using the sublytic membrane attack complex (MAC) (Stratton et al., 2015). Meanwhile, d-pantethine, a derivative of vitamin B5, is needed in the production of coenzyme A. It strongly reduces the total levels of cholesterol in cells via its lipidmetabolizing activity (Kosgodage et al., 2017). It also diminishes the shedding of MVs (Kosgodage et al., 2017). Similarly to bisindolylmaleimide-I, it inhibits the translocation of PS (Martin et al., 2016) and therefore prevents microvesiculation. However, d-pantethine severely reduces cell viability by nearly $80 \%$ in PC3 cells (Kosgodage et al., 2017).

\section{Inhibitors Targeting ATP-Binding Cassette Transporters or Other Proteins Within Cell}

Inhibitors targeting ATP-binding cassette (ABC) transporters include glyburide (glibenclamide) and indomethacin. Glyburide, a drug for diabetes, inhibits the ATP-sensitive $\mathrm{K}^{+}$channel of an $\mathrm{ABC}$ transporter participating in the release of MVs (Catalano and O'driscoll, 2020). Glyburide non-specifically interacts with the SUR receptor (an ABC transporter) and other proteins such as ABCA1, which regulates cellular cholesterol and phospholipid concentrations (Liu and Tang, 2012). Since cholesterol is vital to the release of both MVs and exosomes, glyburide should be able to have both exosome-inhibiting and MV-inhibiting abilities. Even so, Kosgodage et al. (2017) showed that glyburide did 
not affect MV biogenesis in PC3 and MCF-7 cells (Kosgodage et al., 2017) and its effect on exosomes was not tested in the study. In comparison, indomethacin, an anti-inflammatory drug, specifically downregulates the transcription of ABCA3, which aids in the transport of lipids (Aung et al., 2011). This explains why indomethacin was able to act as a chemosensitizer for lymphoma cell lines SU-DHL-4, OCl-Ly1 and OCl-Ly3 (Koch et al., 2016). Prior to treatment with indomethacin, the cells encapsulated chemotherapy drugs doxorubicin and pixantrone in exosomes which were then effluxed from cells (Koch et al., 2016). The application of $10 \mu \mathrm{M}$ indomethacin to the lymphoma cells allowed the drugs to accumulate within their nuclei and exert cytostatic effects (Koch et al., 2016). Although there are many ABC transporter inhibitors such as pelitinib and vatalanib have been identified to sensitize resistant cancer cell lines, e.g., lung and colon cancer cell lines successfully (To et al., 2015a,b), but the study on the effect of these inhibitors in overcoming EV-mediated drug resistance is limited.

Simvastatin, a HMG-CoA reductase inhibitor, prevents the synthesis of cholesterol; since cholesterol is an integral constituent in endosomal membranes, this reduced exosome secretion in epithelial cells and monocytes by $40 \%$ (Kulshreshtha et al., 2019). However, simvastatin has not yet been tested with cancerous cell lines. Simvastatin (but not GW4869) was further shown to decrease intracellular concentrations of proteins associated with exosomes such as ALIX, CD63, and CD81 (Kulshreshtha et al., 2019). Simvastatin also inhibits endocytosis (specifically, CIE) dose-dependently (Costa Verdera et al., 2017).

\section{Inhibitors of Cytoskeletal Organization}

Proteins which participate in cytoskeletal organization are targets for various inhibitors since they are vital for EV release as well as endocytic processes. These proteins may specifically interact with actin; or they may have broad effects on cytoskeleton organization, in which case they are classified as cytoskeletonrelated proteins. The targeting of EV release via these pathways may reduce the transfer of vesicular content between cancer cells and limit the transmission of chemoresistance (Figure 3).

\section{Inhibitors of Actin-Interacting Proteins}

Cytochalasin D is a fungal toxin which binds to actin filament edges, preventing actin polymerization (Catalano and O'driscoll, 2020). Actin polymerization is vital in microvesiculation as well as the trafficking of MVBs toward the cell membrane; thus, cytochalasin D can inhibit EV release. It was proven that HeLa (human cervical adenocarcinoma), Panc 1 (pancreatic carcinoma), PC3, and A293 (human embryonic kidney epithelial) cells secreted exosomes carrying survivin, an anti-apoptotic protein (Khan et al., 2011). It was shown that treatment with cytochalasin D treatment decreased the release of exosomes in those cell lines, therefore reducing the concentration of survivin in the tumor environment (Khan et al., 2011). Cytochalasin D may be capable of inhibiting macropinocytosis, as macropinocytosis also involves actin-dependent pathways (Costa Verdera et al., 2017). However, its effect is not specific in targeting endocytosis mechanisms and its efficacy between different cell lines vary (Dutta and Donaldson, 2012).

\section{Inhibitors Targeting Other Cytoskeleton-Related Proteins}

Calpeptin is currently the most well-characterized calpain inhibitor. Calpains are cytosolic proteases and their activated forms participate in cellular activities including cell cycle progression, cell motility and cytoskeleton remodeling (Jorfi et al., 2015). Calpains are deregulated in cancer cells and thus play a role in cancer progression, apoptosis and migration. As per Figure 3, calpains promote the shedding of MVs through cytoskeletal rearrangement; thus, calpain inhibitors such as calpeptin can reduce the amount of MV shedding by cells, and they reduce cell proliferation as well. Jorfi et al. (2015) studied the chemosensitizing effect of calpeptin inhibition on the PC3 cell line, where the level of cell proliferation and apoptosis due to docetaxel treatment increased substantially in comparison to PC3 cells which did not receive calpeptin (Jorfi et al., 2015).

Chloramidine (Cl-amidine) is a calcium chelator which irreversibly binds to peptidylarginine deiminase (PAD), consequently causing post-translational protein deimination. PAD-modulated deimination of actin in the cytoskeleton is needed in order for MVs to form (Catalano and O'driscoll, 2020). Kosgodage et al. (2017) demonstrated the ability of Cl-amidine to reduce the release of exosomes as well as MVs (Kosgodage et al., 2017), showing the involvement of PAD in exosome release. $\mathrm{Cl}$-amidine may inhibit EV uptake as well. It has been shown to target both CIE and CDE (Vercauteren et al., 2010) although this targeting is unspecific (Dutta and Donaldson, 2012).

Another inhibitor, NSC23766, acts on Rac1, a Rho GTPase which is involved in cytoskeletal remodeling (Catalano and O'driscoll, 2020). In cancer cells, activated Racl (bound to GTP) contributes to chemoresistance by contributing to angiogenesis and by binding to regulators of apoptosis (such as Bcl-2) which arouses anti-apoptotic responses from the cell (Liu et al., 2019). NSC23766 blocks the activation of Rac1 via guanine nucleotide exchange factors (GEF) Trio and Tiam1 (Catalano and O'driscoll, 2020). While NSC23766 has been shown to strongly reduce MV secretion in non-cancerous human cells (Wang et al., 2017), though it does not appear to have a significant effect when used alone in cancer cells without a second EV inhibitor (Wu et al., 2019). It may also function as an inhibitor of macropinocytosis (Hernandez et al., 2010).

Y27632 is a competitive inhibitor of ROCK1 and ROCK2, cytoskeleton-interacting members of the ROCK family (Catalano and O'driscoll, 2020). Y27632 has been used to demonstrate the relation of ROCK1 and ROCK2 to MV formation (Hartmann et al., 2015). ROCK1 and ROCK2 activate LIMK and MYLK (Li et al., 2012), which affect cofilin and myosin to stimulate cytoskeleton re-organization and the contraction of actin filaments (Kosgodage et al., 2017), both activities of which are integral to the process of microvesiculation. Y27632 competes with ATP to bind ROCK1 and ROCK2 catalytic binding sites (Catalano and O'driscoll, 2020). Y27632 acts significantly on MV-sized vesicles, reducing their secretion by $67 \%$ in PC3 cells and decreasing exosome-sized secretion by only $3 \%$ 


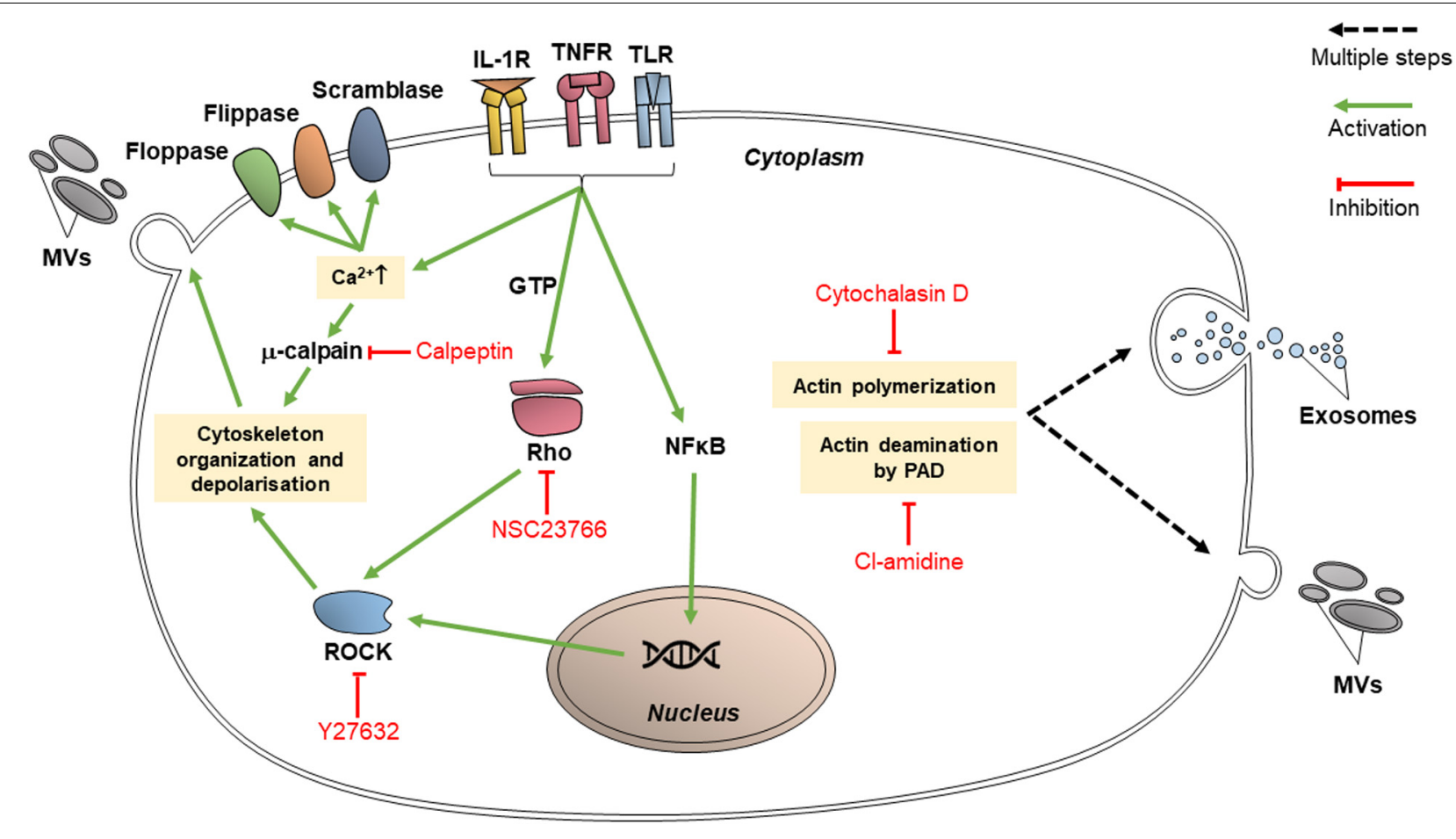

FIGURE 3 | Inhibitors of cytoskeletal organization pathways (calpeptin, Y27632, NSC23766, cytochalasin D, and chloramidine/Cl-amidine) inhibit EV release through intracellular trafficking and budding of the vesicles.

(Kosgodage et al., 2017). In a study by Li et al. (2012), tissue transglutaminase immunohistochemical staining of MDA-MB231 (breast adenocarcinoma), U87 (glioblastoma) and HeLa cells following their treatment with Y27632 showed that MVs were not visible at their surfaces ( $\mathrm{Li}$ et al., 2012) and the medium conditioned by Y27632-treated MDA-MB-231 and U87 cells contained a significantly lower level of MVs compared to the level prior to Y27632 treatment (Li et al., 2012).

\section{Inhibitors of EVs Release}

Extracellular vesicles are released using many other mechanisms such as those involving protein kinases, calcium channels and other molecules, as well as the ESCRT-dependent pathway (Figure 4). These targets for inhibition of EV release may not be directly related to lipid-related pathways or cytoskeleton organization and are hence classified separately. Nonetheless, these pathways are vital in EV release, and their inhibition could decrease the conveyance of chemoresistance between cancer cells.

\section{Inhibitors Targeting Protein Kinases}

These may include Y27632 (described in section "Inhibitors Targeting Other Cytoskeleton-Related Proteins"), U0126, imatinib, and dasatinib. U0126 specifically and noncompetitively inhibits MEK 1 and MEK 2, mitogen-activated protein kinase kinase (MAPKK) protein kinases (Catalano and O'driscoll, 2020), which prevents the activation of ERK, needed for microvesiculation to occur. U0126 is proved to reduce MV secretion (Catalano and O'driscoll, 2020) and when used in combination with gemcitabine for $72 \mathrm{~h}$, increased levels of cell death in chemoresistant Suit-2 cells by 11-fold compared to the control (Becker et al., 2016). Dual treatment of another chemoresistant human pancreatic cancer cell line, MPanc-96, with U0126 and gemcitabine for $72 \mathrm{~h}$ also increased the levels of cell death, though not as dramatically as in Suit-2 (Becker et al., 2016). These results show that U0126 is an effective MV inhibitor able to induce chemosensitivity in chemoresistant cell lines, although its efficacy varies between cell lines.

Imatinib and dasatinib, medications for leukemia, both inhibit ATP-binding sites of the catalytic sites of bcr-abl tyrosine kinase enzymes (Catalano and O'driscoll, 2020). Phosphorylated receptor tyrosine kinases promote anti-apoptotic activity in cancer-derived exosomes (Song et al., 2016). Dasatinib was developed when myeloid leukemia cells were shown to acquire resistance to imatinib, and dasatinib was proven to be effective on all mutants of the bcr-abl tyrosine kinase enzyme (Mineo et al., 2012). Imatinib and dasatinib reduced exosome biogenesis in the chronic myeloid leukemia cell line K562 by 58 and 56\%, respectively (Mineo et al., 2012).

\section{Inhibitors Targeting ESCRT-Dependent Pathway of EVs Production}

These include manumycin A, tipifarnib, and sulphisoxazole. Manumycin A and tipifarnib both diminish levels of exosome production primarily by targeting Ras farnesyltransferase enzymes, hence inhibiting their activation in order for Ras/Raf/ERK1/2 signaling to proceed, which is part of the 


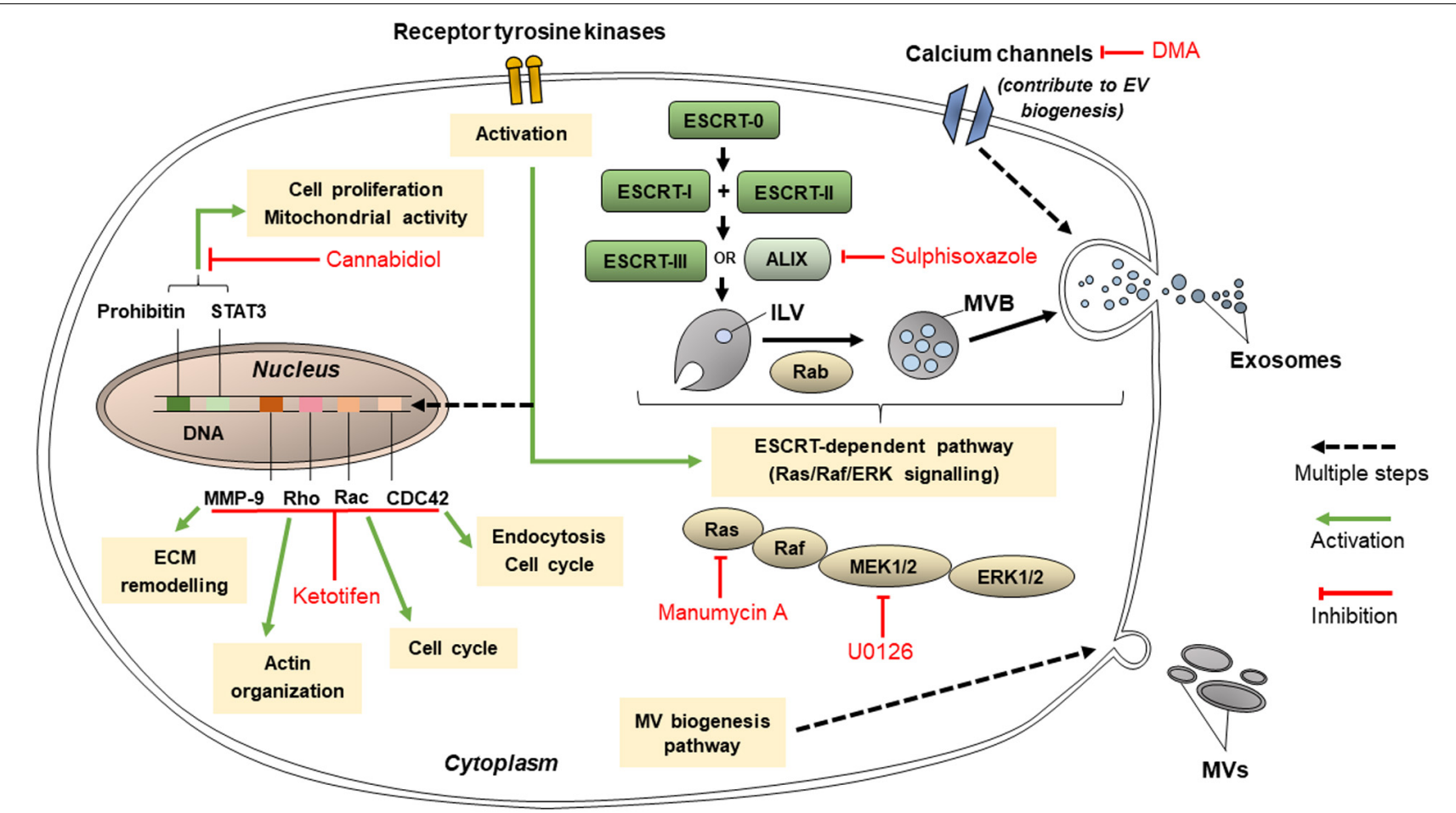

FIGURE 4 | Pathways that targeted by cannabidiol, ketotifen, manumycin A, U0126, sulphisoxazole, and dimethyl amiloride (DMA) for inhibition of EV release.

ESCRT-dependent pathway of exosome biogenesis (Hanson and Cashikar, 2012; Datta et al., 2018). However, manumycin A also inhibits nSMase2 activity (Canals and Hannun, 2013) and hnRNP H1 (Datta et al., 2017). It has been suggested at low concentrations of manumycin A, nSMase 2 inhibition does not occur and that both nSMase 2 and Ras farnesyltransferase activity is needed in order for manumycin A to efficiently inhibit exosome biogenesis (Datta et al., 2017). Manumycin A $(250 \mathrm{~m})$ was found to inhibit exosome biogenesis by 50 , 60 , and $65 \%$ in prostate cancer cell lines C4-2B, PC3, and 22Rv1, respectively (Datta et al., 2018). Meanwhile, tipifarnib activity was found to reduce the phosphorylation of signal transducers and activators of transcription 3 (STAT3) and ERK, the latter of which is needed in exosome biogenesis, and both of which are needed for the growth of cancer cells (Datta et al., 2018). Tipifarnib (1 $\mu \mathrm{M})$ was found to inhibit exosome biogenesis by $70 \%$ in a modified version of human prostate cancer cell line C4-2B which expressed the exosomal marker CD63 (Datta et al., 2018).

Sulphisoxazole are antibacterial drugs which, by targeting endothelin receptor type A (a G-protein coupled receptor), demonstrates inhibitory activity on components within or relating to the ESCRT-dependent pathway such as ALIX and VPS4B (which aids in ILV formation) as well as some RABs (Catalano and O'driscoll, 2020). It was proven to reduce the secretion of small EVs in breast adenocarcinoma cell lines MCF-7, MCF-10A, and MDA-MB-231 (Im et al., 2019). Most of the small EVs inhibited were likely to be exosomes, since sulphisoxazole did not inhibit the secretion of MVs from the breast cancer cell lines used (Im et al., 2019).

\section{Inhibitors Targeting Other Pathways of EVs Release}

Cannabidiol and SMR peptides are recently discovered to be able to inhibit EV release. Cannabidiol, a phytocannabinoid, has been discovered to inhibit EV release from HEPG2 as well as MDA-MB-231 and PC3 cell lines (Kosgodage et al., 2018). Its effect is dose-dependent and varies across different cancer cell types. It has been found to affect mitochondrial functions via reducing the expression of STAT3 and prohibitin, both of which positively regulate cell proliferation, and hence is a possible agent to sensitize chemoresistant cells to chemotherapy drugs (Kosgodage et al., 2018). Meanwhile, SMR peptides impedes mortalin expression (Huang et al., 2019). Mortalin is present in erythroleukemia K562 cell-derived EVs and has been implicated in $\mathrm{EV}$ release, although its precise relation is not known (Pilzer and Fishelson, 2005). SMR peptides blocked EV release in the cell lines MCF-7 and MDA-MB-231 (Huang et al., 2019). It inhibited the growth of the breast cancer cells without affecting their cell viabilities (Huang et al., 2019).

Ketotifen (an antihistamine) and dimethyl amiloride (DMA) (a derivative of amiloride, a drug to treat high blood pressure) inhibit exosome release by altering intracellular calcium levels (Khan et al., 2018; Catalano and O'driscoll, 2020). Calciumdependent pathways are prevalent in exosome release (Khan et al., 2018). Ketotifen and DMA hinder calcium entry into cells. DMA targets calcium channels (Catalano and O'driscoll, 2020) whereas ketotifen inhibits the expression of CDC42, Rac, 
Rho and MMP-9 (Kim et al., 2014) as indicated in Figure 4. Ketotifen sensitized HeLa cells, as well as MCF-7 and BT549 breast cancer cells, to doxorubicin proportionally to its effect on exosome release inhibition (Khan et al., 2018). DMA was shown to decrease exosome secretion both in vitro and in vivo (Chalmin et al., 2010). An in vitro study using CT26 (mouse colon carcinoma), EL4 (mouse lymphoma) and H23 (human lung adenocarcinoma) cell lines showed that DMA reduced exosome release as per analysis of the culture medium (Chalmin et al., 2010). In vivo results of the study are discussed in section "Targeting EVs in Clinical Applications." Additionally, as calcium channels are also involved in micropinocytosis, DMA has been shown to inhibit macropinocytosis in mammalian cells, though it has not yet been used in cancer cell lines specifically.

\section{Inhibitors of Endocytosis}

Pathways of clathrin-dependent and CIE may be targeted by $\mathrm{EV}$ inhibitors to prevent the uptake of EVs by cancer cells (Figure 5). If EV uptake inhibitors are utilized, even if EVs are released by chemoresistant cancer cells, the uptake of those EVs by chemosensitive cells will be reduced, allowing those cells to remain sensitive to chemotherapy drugs.

\section{Inhibitors of CDE}

Choosing the appropriate inhibitor to investigate a specific endocytic pathway can be challenging as they may be unspecific or toxic for different cell lines. Inhibitors of CDE include dynasore and ikarugamycin (IKA) as well as older inhibitors such as chlorpromazine, potassium depletion and hypertonic sucrose (Dutta and Donaldson, 2012). While potassium depletion and hypertonic sucrose are still commonly used to study CDE, they are non-specific as they may target CIE as well as CDE (Dutta and Donaldson, 2012). Potassium depletion may result in decreases of protein and DNA synthesis, whereas hypertonic sucrose results in shrinkage of cells and could affect cytoskeletal actin. Chlorpromazine blocks CDE effectively by assembling clathrin and adaptor proteins on endosomal membranes, depleting plasma membranes of clathrin (Vercauteren et al., 2010). It has also targets CIE and was discovered to be toxic to several noncancerous cell lines (Vercauteren et al., 2010).

Dynasore and IKA are more recent inhibitors. Dynasore is a widely used CDE inhibitor which has been thoroughly characterized. But like the other CDE inhibitors, dynasore too may have non-specific effects. Dynasore non-competitively inhibits GTPase activity of the dynamin proteins dynamin1, dynamin2 and Drp1 (mitochondrial dynamin) in just seconds. Dynamin proteins are necessary for a late step of CDE which involves the production of a clathrin-coated endocytic vesicle (as indicated in Figure 5) and may participate in earlier stages of CDE (Kirchhausen et al., 2008). Dynasore inhibits the endocytosis of exosomes (Dutta and Donaldson, 2012). Dynasore may not only act as a CDE inhibitor: previous studies have shown that dynamin proteins regulate the assembly of actin filaments into bundles ( $\mathrm{Gu}$ et al., 2010). Hence, it could be hypothesized that dynasore could have some additional effect on exosome release in cells. However, this has not been tested in cancer cells and this hypothesis is dubious since dynasore was shown to be ineffective in inhibiting exocytosis in synaptic vesicles (Newton et al., 2006). In comparison, IKA has been found to exclusively inhibit CDE in H1299, HCC366 and H1437 lung cancer cell lines (Elkin et al., 2016). It targets different receptors involved in CDE according to the cell line. In H1299 cells, it acts on transferrin receptors (TfNR) to inhibit CME (Elkin et al., 2016). $4 \mu \mathrm{M}$ of IKA was also found to have no significant effect on cell viability of $\mathrm{H} 1299$ for the first 8 h; after 48 h, significant levels of apoptosis occurred and only $20 \%$ of the cells remained viable (Elkin et al., 2016). At $32 \mu \mathrm{M}$ of IKA, the concentration of IKA which absolutely inhibits CDE, reductions in cell viability of H1299 were detected within an hour of treatment with IKA and after $8 \mathrm{~h}$ of incubation, only $20 \%$ of cells remained viable (Elkin et al., 2016). The cytotoxicity of IKA with other cancerous cell lines has not been thoroughly examined. The efficacy, specificity and toxicity of CDE inhibitors may be highly variable between different cell lines and suitable inhibitors must be chosen carefully for various types of research.

\section{Inhibitors of CIE}

Caveolin-mediated endocytosis (CavME) inhibitors include genistein and methyl- $\beta$-cyclodextrin $(\mathrm{M} \beta \mathrm{CD})$ as well as heparin. Genistein is a highly specific, dose-dependent inhibitor of tyrosine kinases such as the EGF receptor kinase, and pp60 $60^{v-a r c}$ and pplloBngag-fes kinases (Costa Verdera et al., 2017). It disrupts the arrangements of actin and prevents the mobilization of dynamin for plasma membranes, two mechanisms vital for CIE (Costa Verdera et al., 2017). Genistein has been reported to be toxic to ovarian tumor cells A2780, CaOV3, ES2, and SK-OV-3 at concentrations above $10 \mu \mathrm{M}$ (Antosiak et al., 2017). Meanwhile, M $\beta C D$ removes cholesterol from the plasma membrane, disturbing lipid rafts within it, subsequently diminishing the uptake of exosome-sized vesicles $(\leq 150 \mathrm{~nm})$ (Kosgodage et al., 2017). However, its effect is non-specific as it also inhibits exosome secretion from cells as well as other endocytic pathways such as macropinocytosis and CDE (Dutta and Donaldson, 2012). In the study by Kosgodage et al. (2017), M $\beta C D$ reduced exosome secretion by $58 \%$ compared to the control. $\mathrm{M} \beta \mathrm{CD}$ has been documented to severely impair cell morphology especially under incubation times which are longer than $2 \mathrm{~h}$, using concentrations of $\mathrm{M} \beta \mathrm{CD}$ higher than $5 \mathrm{mM}$, which is in the range of concentrations of $\mathrm{M} \beta \mathrm{CD}$ typically used to inhibit exocytosis (Kosgodage et al., 2017). M $\beta C D$ is typically used in combination with lovastatin, an inhibitor of cholesterol synthesis, to prevent cholesterol being synthesized in cells and replacing the cholesterol in the plasma membrane extracted by $\mathrm{M} \beta \mathrm{CD}$ (Kosgodage et al., 2017).

Heparin, an anticoagulant, competitively inhibits cancer cell surface receptors which depend on heparin sulfate proteoglycan (HSPG) coreceptors for the uptake of exosomes when HSPGs are present (Sento et al., 2016). An example of a HSPG which is necessary for exosome biogenesis is Syndecan (Sento et al., 2016). Heparin primarily acts on the receptors of treated cells rather than the receptors on treated exosomes, as shown by a study using the urothelial carcinoma cell line SW780 (Franzen et al., 2014). In another study by Christianson et al. (2013), it 


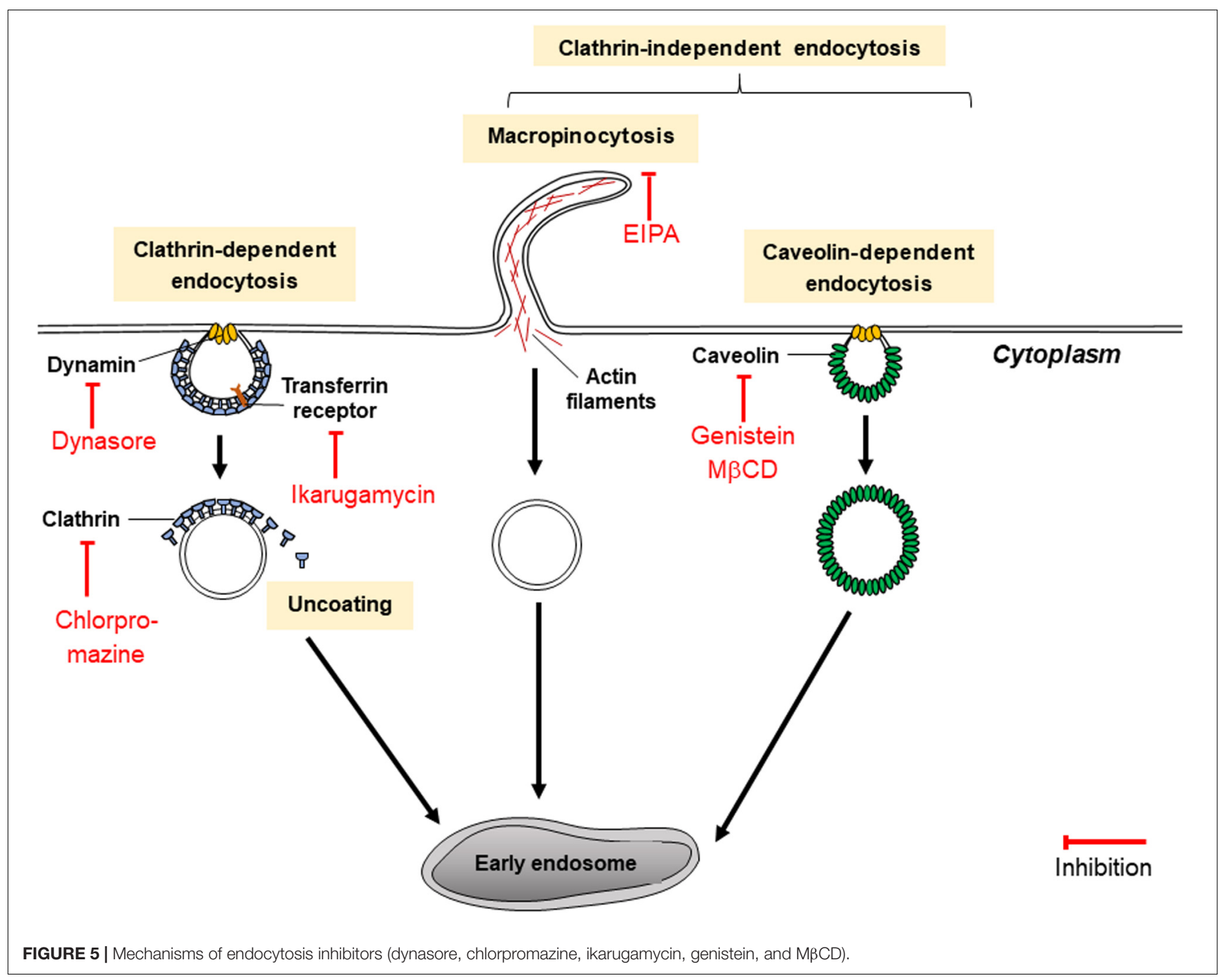

was observed that exosome uptake in U87 cells was reduced by $55 \%$ compared to the untreated control. In addition, exosome uptake was reduced significantly in medium depleted of $\mathrm{Ca}^{2+}$ compared to medium consisting $\mathrm{Ca}^{2+}$ (Christianson et al., 2013). It was concluded that heparin inhibits exosome internalization in a dose- and charge density-dependent way (Christianson et al., 2013).

5-(N-ethyl-N-isopropyl)amirolide (EIPA) is one chemical derivative of amiloride which has been regularly used to inhibit macropinocytosis. EIPA lowers sub-membranous $\mathrm{pH}$ by targeting $\mathrm{Na}^{+} / \mathrm{H}^{+}$exchangers (Koivusalo et al., 2010), and like NSC23766, disrupts Rac1 activation as well as the assembly of actin, hence reducing the uptake of EVs as demonstrated using HeLa cells (Costa Verdera et al., 2017). While EIPA is regarded as effective as a pharological inhibitor of macropinocytosis, it inflicts "collateral damage" on treated cells with effects on ion transport, intracellular $\mathrm{pH}$ and the cytoskeleton (Costa Verdera et al., 2017). Imipramine has been suggested as an alternative; intracellular $\mathrm{pH}$ of treated cells has been proven to recover following treatment with imipramine, and imipramine was shown to effectively reduce macropinocytosis activity in a range of cell lines including mammary carcinoma 4T1 cells (Lin et al., 2018).

\section{Difficulties, Limitations, and Considerations in EVs Inhibition}

The challenges in using EV-inhibiting drugs to tackle chemoresistance relate to the efficacy, specificity and toxicity of inhibitors; lack of knowledge regarding the precise mechanism of several inhibitors; and adapting functional and potent inhibitor drugs for clinical use. These challenges are further complicated by still-evolving knowledge regarding EV-related pathways.

First, many EV inhibitors vary in their efficacy (between different cell lines and cell types) and their specificity. In a clinical setting, this could lead to difficulty in choosing an appropriately efficacious inhibitor for treatment. Some EV inhibitors may be so specific as to target a single protein involved in EV biogenesis or uptake such as indomethacin which targets ABCA3; but some may have a wide range of targets with varying downstream effects, such as manumycin A which may target Ras farnesyltransferase 
and nSMase2 enzymes. Some EV inhibitors may target both exosomes and MVs (such as cannabidiol, Cl-amidine, and imipramine), whereas others (such as calpeptin, cytochalasin D, and Y27632) may target the exocytosis of only one subtype of $\mathrm{EV}$. Some EV inhibitors may even target pathways of EV release as well as those of EV uptake, since those pathways may share similar mechanisms, for instance cytochalasin $D, M \beta C D$ and simvastatin. A particular inhibitor may inhibit the release of certain EV populations but promote the release of others. For example, GW4869 inhibits exosome release but was reported to stimulate MV release in breast adenocarcinoma SKBR3 cells (Menck et al., 2017).

Many EV inhibitors do not completely inhibit EV release or uptake when used independently. This is usually because the activity of each EV-related pathway contributes only a proportion of total EV secretion or release, and most inhibitor drugs do not inhibit all the pathways of EV release or EV uptake with equal efficacy. All EVs secreted by a cell may have been the result of various EV release pathways, and all EVs taken up by a cell may have been ingested by any endocytosis or macropinocytosis pathway. For example, a study by Costa Verdera et al. (2017) reported that genistein and EIPA, inhibitors of CIE and MP, respectively, individually inhibited total EV uptake in A431 cells and HeLa cells by only about 50\%; when genistein and EIPA were used in combination, EV uptake was almost totally suppressed, which suggested that both CIE and MP are major contributors to EV uptake (Costa Verdera et al., 2017). Similarly, in a study by Datta et al. (2017), exosome production reduction levels in prostate cancer cell lines (PC3 and 22Rv1) ranged from 50 to $60 \%$ using $250 \mathrm{~nm}$ of manumycin $\mathrm{A}$, but those levels rose significantly when $10 \mu \mathrm{M}$ of GW4869 was used together (Datta et al., 2017). The efficacy of using both GW4869 and manumycin A, nSMase2 (or ESCRT-independent) and ESCRTdependent inhibitors respectively, in the study proved that both ESCRT-independent and ESCRT-dependent activities are vital in exosome biogenesis in prostate cancer cell lines. Therefore, in order to completely inhibit a subpopulation of EVs, at least two inhibitors should be used.

In clinical settings, the prospect of using two EV inhibitors together with chemotherapy is bound to raise questions regarding managing the side effects of this treatment approach. The relative cytotoxicity of different EV inhibitors to different cell lines may also raise hurdles in research as cytotoxic effects may distort experimental results. EV inhibitors may be toxic at the same concentration found to be optimum for EV inhibition, especially after prolonged incubation. One example of such an inhibitor is IKA, which already reduces cell viability within an hour of treatment and impairs the morphologies of cells and cell organelles under prolonged incubation (Elkin et al., 2016). Another toxic effect of EV inhibitors may include the inhibition of EVs essential to regulate healthy physiological processes in cells unrelated to chemoresistance (Maacha et al., 2019). Since EV inhibitors used should not introduce additional effects unrelated to the pathway under study, the cytotoxic effects of some inhibitors pose some challenge to research. In addition, it has been noted by Dos Santos et al. (2011) that, regardless of their toxicity, any endocytosis inhibitors chosen (e.g., chlorpromazine and genistein) should not cause reorganization of the cytoskeleton as this may affect EV uptake mechanisms unrelated to actin, thus distorting the functions of plasma membrane proteins (Santos et al., 2018). This may obfuscate experimental results. As a treatment approach, usage of EV inhibitors which are toxic to normal cells may eventually lead to severe damage to organs.

While there may not be a perfect inhibitor yet, the pursuit to discover fast-acting inhibitors which have specific, reversible and minimally toxic effects should continue (Dutta and Donaldson, 2012). For the study of endocytic and exocytic mechanisms related to chemoresistance in cancer cells, using EV inhibitors alone may not be sufficient, since EV inhibitors may have low specificities. A possible complement to this research for the confirmation of results is the knockdown of proteins and regulators (Costa Verdera et al., 2017) associated with EVrelated pathways such as ALIX, Vsp4 and Rab proteins for exosome biogenesis; aSMase, cholesterol, ARF6 and ARF1 for microvesiculation; dynamin-1, dynamin-2, and adaptor protein2 (a major coat protein) for CDE (Mettlen et al., 2018); ARF6, CDC42, RhoA and caveolin-1 for CIE; and Rac1 for macropinocytosis.

\section{TARGETING EVs IN CLINICAL APPLICATIONS}

At this stage, most drug inhibitors of EV release or uptake have only been tested in the context of chemoresistance under in vitro conditions using various cancer cell lines. However, DMA was assessed in a pre-clinical study by Chalmin et al. (2010) to determine their ability to curb exosome release in vivo. In vivo, DMA reduced exosome release into the bloodstream of mice carrying tumors (Chalmin et al., 2010). Combination therapy of tumor-carrying mice with DMA and the chemotherapy drug cyclophosphamide staunched tumor growth by $50 \%$ or more compared to the untreated controls (Chalmin et al., 2010). These results were consistent among the three mousetumor models created using two mouse cancer cell lines, EL4 and TS/A, and one human cancer cell line, CT26. Reduced tumor growth was attributed to DMA sensitizing the tumor cells to cyclophosphamide. As such, DMA holds potential in improving the efficacy of chemotherapy treatment. More preclinical research involving EV inhibitors discussed in this review is required prior to establishing the ability of EV inhibitors to mitigate chemoresistance in a clinical context.

Alternatively, EVs may be targeted using a genetic approach. For example, knockdown of the nSMase2 gene suppressed EV release and inhibited angiogenesis and metastasis in a xenograft mouse model of breast cancer (Kosaka et al., 2013). However, nSMase2 is essential to intercellular communication in all cells, including healthy cells. Like EV inhibition, in clinical settings, the knockdown of nSMase 2 may also adversely affect the physiological activities of non-cancerous cells. In addition, nSMase2 knockdown failed to impact EV synthesis and release in the prostate cancer cell line PC3 (Phuyal et al., 2014). Prior to clinical implementation of this approach, other genes related 
to EV production in specific cancer types should be analyzed as potential targets. Bobrie et al. (2012) also utilized short hairpin RNA (shRNA) specific to the Rab27a gene to silence its expression, after establishing that Rab27a is involved in exosome secretion (Bobrie et al., 2012). The results of Rab27a inhibition varied between the two breast cancer cell lines used, 4T1 and TS/A. Using a mouse model, it was shown that shRNA targeting Rab27a decreased growth and metastasis of $4 \mathrm{~T} 1$ whereas the same targeting did not affect growth or metastasis of TS/A. The study by Bobrie et al. (2012) highlights the importance of specific treatments for different types of cancer cells; even two cell lines derived from the same tissue can respond very differently to a treatment.

Rather than targeting EV biogenesis or uptake, EVs may also be removed from the circulation. Marleau et al. (2012) strategized a hemofiltration system targeting breast cancer-derived EVs which expressed human epidermal growth factor receptor type 2 (HER2). EVs expressing HER2 were found to stimulate tumor growth and induce the formation of premetastatic niches, promoting metastasis (Marleau et al., 2012). HER2-expressing EVs promote chemoresistance and reduce the effectiveness of breast cancer treatments (Marleau et al., 2012) so elimination of these EVs may prevent metastatic formations. This treatment strategy holds promise, but it is specific to breast cancer. For other types of cancers, different receptors expressed by EVs secreted by specific kinds of cancers should be investigated as potential targets in the hemofiltration system strategy.

In another study, antibodies to CD9 and CD63, glycoprotein receptors present on the surface of EVs, were used to deplete
EVs in a xenograft mouse model of breast cancer (NishidaAoki et al., 2017). The study yielded a pronounced reduction in metastasis, especially for treatment using anti-CD63 antibodies. However, unlike the hemofiltration system described by Marleau et al. (2012), this procedure does not specifically target only cancer-derived EVs. EVs secreted by normal body cells will also be targeted, preventing intercellular communication between healthy cells and adversely affecting regular cellular activities within the body. This treatment also did not impact the growth of the primary tumor site. In the future, the effect of combined treatments of anti-CD63 and chemotherapy drugs on animal breast cancer models could be investigated to reduce primary tumor growth while decreasing metastatic activity. In addition, antibodies targeting receptors more specific to breast cancer (and other types of cancers) can be used instead to specifically target cancer-derived EVs. This will reduce the side effects of this treatment strategy in clinical settings.

Extracellular vesicle inhibition, control of EV-related gene expression and hemofiltration of EVs all prevent or reduce intercellular communication between cancer cells. When these methods are combined with chemotherapy, they can be expected to result in the repression of EV-mediated mechanisms of chemoresistance such as EMT (Figure 1). Chemoresistance is linked to metastasis and tumor relapse (D'alterio et al., 2020), two main causes of deaths among cancer patients (Avanzini and Antal, 2019; Dillekås et al., 2019). Thus, suppressing EV-mediated chemoresistance can be speculated to reduce rates of metastasis and tumor relapse among cancer patients receiving chemotherapy.

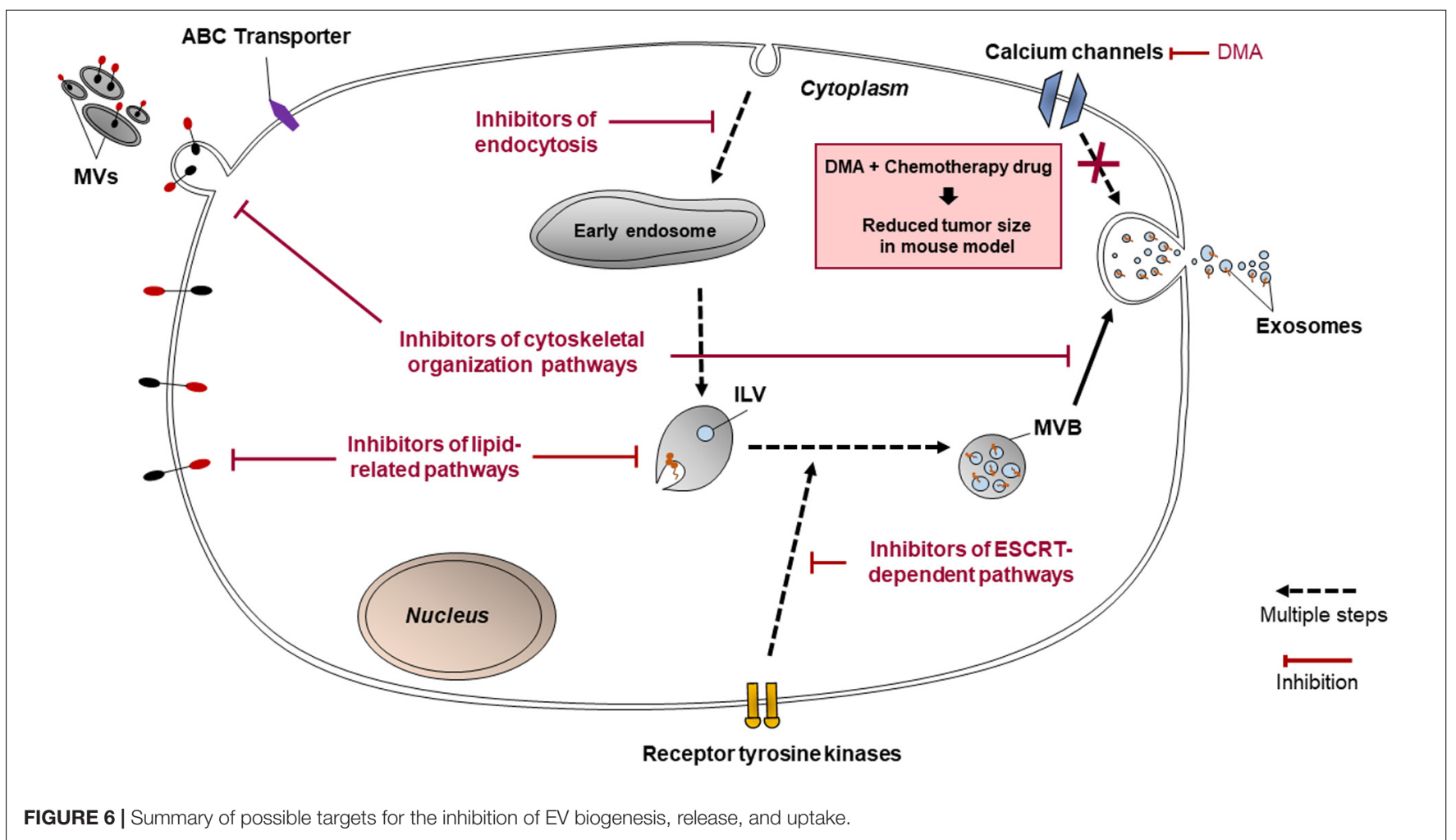


Clinical progresses in targeting EV-mediated chemoresistance are slowed by gaps in knowledge regarding the roles of EVs in mechanisms of chemoresistance. Considering this, investigations into existing drugs which inhibit the release or uptake of EVs may yield discoveries regarding their suitability for use in cancer treatments. Their suitability for use may be defined in terms of their efficacy and toxicity when used alongside chemotherapy drugs. Further research into novel methods to target EV-mediated intercellular communication between cancer cells may aid in planning anticancer therapies while minimizing side effects.

\section{CONCLUSION}

Exosomes and MVs contribute significantly to chemoresistance in many cancer cell lines via mechanisms such as the transfer of miRNAs, which activate anti-apoptotic signaling and DDR, and by enhancing other processes such as chemotherapy drug efflux, immunosuppression, alteration of cytosolic $\mathrm{pH}$ and EMT. Hence, the inhibition of EV release and uptake could be a promising companion treatment to enhance the efficacy of chemotherapy drugs. Since both exosomes and MVs can be released and taken up by cancer cells via multiple pathways, and since those pathways involve diverse and specific mechanisms, there are many possible targets for the inhibition of $\mathrm{EV}$ release and uptake. Such targets may include proteins, transporters, protein kinases and enzymes involved in lipid-related pathways of EV release, cytoskeletal organization, the ESCRT-dependent pathway of EV release, clathrin-dependent and clathrin-independent endocytosis (summarized in Figure 6).

Extracellular vesicle inhibitors may have the capacity to sensitize chemoresistant cells under both in vivo and in vitro conditions; in the case of DMA, this has already been proven using a mouse model. Yet, this strategy requires much investigation as preclinical studies of chemoresistance using EV

\section{REFERENCES}

Abels, E. R., and Breakefield, X. O. (2016). Introduction to Extracellular Vesicles: Biogenesis, RNA Cargo Selection, Content, Release, and Uptake. Cell. Mole. Neurobiol. 36, 301-312. doi: 10.1007/s10571-016-0366-z

Antosiak, A., Milowska, K., Maczynska, K., Rozalska, S., and Gabryelak, T. (2017). Cytotoxic activity of genistein-8-C-glucoside form Lupinus luteus L. and genistein against human SK-OV-3 ovarian carcinoma cell line. Med. Chem. Res. 26, 64-73. doi: 10.1007/s00044-016-1725-5

Arii, J., Watanabe, M., Maeda, F., Tokai-Nishizumi, N., Chihara, T., Miura, M., et al. (2018). ESCRT-III mediates budding across the inner nuclear membrane and regulates its integrity. Nat. Commun. 9, 3379-3379.

Aung, T., Chapuy, B., Vogel, D., Wenzel, D., Oppermann, M., Lahmann, M., et al. (2011). Exosomal evasion of humoral immunotherapy in aggressive B-cell lymphoma modulated by ATP-binding cassette transporter A3. Proc. Natl. Acad. Sci. U S A 108, 15336-15341. doi: $10.1073 /$ pnas. 1102855108

Avanzini, S., and Antal, T. (2019). Cancer recurrence times from a branching process model. PLoS Comput. Biol. 15:e1007423-e1007423. doi: 10.1371/ journal.pcbi. 1007423

Awojoodu, A. O., Keegan, P. M., Lane, A. R., Zhang, Y., Lynch, K. R., Platt, M. O., et al. (2014). Acid sphingomyelinase is activated in sickle cell erythrocytes inhibitors are limited. Pathways related to EV biogenesis, release and uptake mechanisms have yet to be thoroughly explained, and the mechanisms of existing EV inhibitors must be studied further, especially for the use of inhibitors in combination to fully block all avenues of EV production or uptake. More efficacious EV-inhibiting drugs must be developed before they can be implemented in a clinical setting. Further investigation into EV release and uptake pathways is required in order to fully describe the mechanisms involved, and to develop more advanced EV inhibitors capable of targeting those specific mechanisms to counteract chemoresistance. This is vital in advancing the effectiveness of anticancer treatments.

\section{AUTHOR CONTRIBUTIONS}

$\mathrm{RH}$ performed searching, analyzed data, and wrote the manuscript. NK, B-HG, and W-LL contributed to the conceptual idea, reviewed the drafts, supervised the writing process, and provided important information for the completion of this manuscript. LM and ZF reviewed the drafts and refined the manuscript. All the authors revised and approved the final version of the manuscript.

\section{FUNDING}

This work was supported by the Fundamental Research Grant Scheme grants (FRGS/1/2019/SKK08/MUSM/02/4) from the Ministry of Higher Education Malaysia.

\section{ACKNOWLEDGMENTS}

Special thanks to Prof. Ian Paterson from the University of Malaya, Malaysia for his generous help in English editing of the article.

and contributes to inflammatory microparticle generation in SCD. Blood 124, 1941-1950. doi: 10.1182/blood-2014-01-543652

Babst, M. (2011). MVB vesicle formation: ESCRT-dependent, ESCRT-independent and everything in between. Curr. Opin. Cell Biol. 23, 452-457. doi: 10.1016/j. ceb.2011.04.008

Becker, A., Thakur, B. K., Weiss, J. M., Kim, H. S., Peinado, H., and Lyden, D. (2016). Extracellular vesicles in cancer: cell-to-cell mediators of metastasis. Cancer Cell 30, 836-848. doi: 10.1016/j.ccell.2016.10.009

Bianco, F., Perrotta, C., Novellino, L., Francolini, M., Riganti, L., Menna, E., et al. (2009). Acid sphingomyelinase activity triggers microparticle release from glial cells. EMBO J. 28, 1043-1054. doi: 10.1038/emboj.2009.45

Binenbaum, Y., Fridman, E., Yaari, Z., Milman, N., Schroeder, A., Ben David, G., et al. (2018). Transfer of miRNA in Macrophage-Derived Exosomes Induces Drug Resistance in Pancreatic Adenocarcinoma. Cancer Res. 78, 5287-5299. doi: 10.1158/0008-5472.can-18-0124

Bobrie, A., Krumeich, S., Reyal, F., Recchi, C., Moita, L. F., Seabra, M. C., et al. (2012). Rab27a supports exosome-dependent and -independent mechanisms that modify the tumor microenvironment and can promote tumor progression. Cancer Res. 72, 4920-4930. doi: 10.1158/0008-5472.can-12-0925

Canals, D., and Hannun, Y. A. (2013). Novel chemotherapeutic drugs in sphingolipid cancer research. Handbook Exp. Pharmacol. 2013, 211-238. doi: 10.1007/978-3-7091-1368-4_12 
Cao, Y. L., Zhuang, T., Xing, B. H., Li, N., and Li, Q. (2017). Exosomal DNMT1 mediates cisplatin resistance in ovarian cancer. Cell Biochem. Funct. 35, 296303. doi: $10.1002 /$ cbf.3276

Catalano, M., and O'driscoll, L. (2020). Inhibiting extracellular vesicles formation and release: a review of EV inhibitors. J. Extrac. Vesicles 9:1703244. doi: 10. 1080/20013078.2019.1703244

Cereghetti, D. M., and Lee, P. P. (2014). Tumor-Derived Exosomes Contain microRNAs with Immunological Function: Implications for a Novel Immunosuppression Mechanism. Microrna 2, 194-204. doi: 10.2174/22115366113029990012

Chalmin, F., Ladoire, S., Mignot, G., Vincent, J., Bruchard, M., Remy-Martin, J.P., et al. (2010). Membrane-associated Hsp72 from tumor-derived exosomes mediates STAT3-dependent immunosuppressive function of mouse and human myeloid-derived suppressor cells. J. Clin. Invest. 120, 457-471.

Chen, Q., Takada, R., Noda, C., Kobayashi, S., and Takada, S. (2016). Different populations of Wnt-containing vesicles are individually released from polarized epithelial cells. Sci. Rep. 6, 35562-35562.

Chorowala, M., Oza, P., and Shah, G. (2012). Mechanisms of anticancer drugs resistance: an overview. Int. J. Pharmaceut. Sci. Drug Res. 4, 1-9.

Christianson, H. C., Svensson, K. J., Van Kuppevelt, T. H., Li, J.-P., and Belting, M. (2013). Cancer cell exosomes depend on cell-surface heparan sulfate proteoglycans for their internalization and functional activity. Proc. Natl. Acad. Sci. 110:17380. doi: 10.1073/pnas.1304266110

Ciardiello, C., Migliorino, R., Leone, A., and Budillon, A. (2020). Large extracellular vesicles: Size matters in tumor progression. Cytokine Growth Fact. Rev. 51, 69-74. doi: 10.1016/j.cytogfr.2019.12.007

Costa Verdera, H., Gitz-Francois, J. J., Schiffelers, R. M., and Vader, P. (2017). Cellular uptake of extracellular vesicles is mediated by clathrin-independent endocytosis and macropinocytosis. J. Control. Release 266, 100-108. doi: 10. 1016/j.jconrel.2017.09.019

D’alterio, C., Scala, S., Sozzi, G., Roz, L., and Bertolini, G. (2020). Paradoxical effects of chemotherapy on tumor relapse and metastasis promotion. Semin. Cancer Biol. 60, 351-361. doi: 10.1016/j.semcancer.2019.08.019

Datta, A., Kim, H., Lal, M., Mcgee, L., Johnson, A., Moustafa, A. A., et al. (2017). Manumycin A suppresses exosome biogenesis and secretion via targeted inhibition of Ras/Raf/ERK1/2 signaling and hnRNP $\mathrm{H} 1$ in castration-resistant prostate cancer cells. Cancer Lett. 408, 73-81. doi: 10.1016/j.canlet.2017.08.020

Datta, A., Kim, H., Mcgee, L., Johnson, A. E., Talwar, S., Marugan, J., et al. (2018). High-throughput screening identified selective inhibitors of exosome biogenesis and secretion: A drug repurposing strategy for advanced cancer. Sci. Rep. 8, 8161-8161.

Dillekås, H., Rogers, M. S., and Straume, O. (2019). Are $90 \%$ of deaths from cancer caused by metastases? Cancer Med. 8, 5574-5576. doi: 10.1002/cam4.2474

Dos Santos, T., Varela, J., Lynch, I., Salvati, A., and Dawson, K.A. (2011). Effects of transport inhibitors on the cellular uptake of carboxylated polystyrene nanoparticles in different cell lines. PloS One 6, e24438-e24438. doi: 10.1371/ journal.pone.0024438

Doyle, L. M., and Wang, M. Z. (2019). Overview of Extracellular Vesicles, Their Origin, Composition, Purpose, and Methods for Exosome Isolation and Analysis. Cells 8:727. doi: 10.3390/cells8070727

Dutta, D., and Donaldson, J. G. (2012). Search for inhibitors of endocytosis: Intended specificity and unintended consequences. Cell. Logist. 2, 203-208. doi: $10.4161 / \mathrm{cl} .23967$

Elkin, S. R., Oswald, N. W., Reed, D. K., Mettlen, M., Macmillan, J. B., and Schmid, S. L. (2016). Ikarugamycin: A Natural Product Inhibitor of Clathrin-Mediated Endocytosis. Traffic 17, 1139-1149. doi: 10.1111/tra. 12425

Fan, J., Wei, Q., Koay, E. J., Liu, Y., Ning, B., Bernard, P. W., et al. (2018). Chemoresistance Transmission via Exosome-Mediated EphA2 Transfer in Pancreatic Cancer. Theranostics 8:5986. doi: 10.7150/thno.26650

Fang, T., Lv, H., Lv, G., Li, T., Wang, C., Han, Q., et al. (2018). Tumor-derived exosomal miR-1247-3p induces cancer-associated fibroblast activation to foster lung metastasis of liver cancer. Nat. Commun. 9:191.

Fang, Y., Zhou, W., Rong, Y., Kuang, T., Xu, X., Wu, W., et al. (2019). Exosomal miRNA-106b from cancer-associated fibroblast promotes gemcitabine resistance in pancreatic cancer. Exp. Cell Res. 383:111543. doi: 10.1016/j.yexcr.2019.111543
Figuera-Losada, M., Stathis, M., Dorskind, J. M., Thomas, A. G., Bandaru, V. V. R., Yoo, S.-W., et al. (2015). Cambinol, a Novel Inhibitor of Neutral Sphingomyelinase 2 Shows Neuroprotective Properties. PLoS One 10:e0124481.doi: 10.1371/journal.pone.0124481

Franzen, C. A., Simms, P. E., Van Huis, A. F., Foreman, K. E., Kuo, P. C., and Gupta, G. N. (2014). Characterization of uptake and internalization of exosomes by bladder cancer cells.(Research Article)(Report). BioMed. Res. Int. 2014:619829.

Gu, C., Yaddanapudi, S., Weins, A., Osborn, T., Reiser, J., Pollak, M., et al. (2010). Direct dynamin-actin interactions regulate the actin cytoskeleton. EMBO J. 29, 3593-3606. doi: 10.1038/emboj.2010.249

Hanson, P. I., and Cashikar, A. (2012). Multivesicular Body Morphogenesis. Annu. Rev. Cell Dev. Biol. 28, 337-362. doi: 10.1146/annurev-cellbio-092910-154152

Hartmann, S., Ridley, A., and Lutz, S. (2015). The Function of Rho-Associated Kinases ROCK1 and ROCK2 in the Pathogenesis of Cardiovascular Disease. Front. Pharmacol. 6:276. doi: 10.3389/fphar.2015.00276

Hazan-Halevy, I., Rosenblum, D., Weinstein, S., Bairey, O., Raanani, P., and Peer, D. (2015). Cell-specific uptake of mantle cell lymphoma-derived exosomes by malignant and non-malignant B-lymphocytes. Cancer Lett. 364, 59-69. doi: 10.1016/j.canlet.2015.04.026

Heltweg, B., Gatbonton, T., Schuler, A. D., Posakony, J., Li, H., Goehle, S., et al. (2006). Antitumor activity of a small-molecule inhibitor of human silent information regulator 2 enzymes. Cancer Res. 66:4368. doi: 10.1158/0008-5472. can-05-3617

Hernandez, E., De La Mota-Peynadot, A., Dharmawardhane, S., and Vlaar, C. P. (2010). Novel inhibitors of Racl in metastatic breast cancer.(Report). Puerto Rico Health Sci. J. 29:348.

Hessvik, N. P., and Llorente, A. (2018). Current knowledge on exosome biogenesis and release. Cell. Mole. Life Sci. 75, 193-208. doi: 10.1007/s00018-017-2595-9

Hetzenecker, S., Helenius, A., and Krzyzaniak, M. A. (2016). HCMV Induces Macropinocytosis for Host Cell Entry in Fibroblasts. Traffic 17, 351-368. doi: 10.1111/tra.12355

Hoehn, R. S., Jernigan, P. L., Japtok, L., Chang, A. L., Midura, E. F., Caldwell, C. C., et al. (2017). Acid Sphingomyelinase Inhibition in Stored Erythrocytes Reduces Transfusion-Associated Lung Inflammation. Ann. Surg. 265, 218-226. doi: 10.1097/sla.0000000000001648

Hu, Y., Yan, C., Mu, L., Huang, K., Li, X., Tao, D., et al. (2015). Fibroblast-Derived Exosomes Contribute to Chemoresistance through Priming Cancer Stem Cells in Colorectal Cancer. PLoS One 10:e0125625-e0125625. doi: 10.1371/journal. pone. 0125625

Huang, M.-B., Wu, J. Y., Lillard, J., and Bond, V. C. (2019). SMR peptide antagonizes mortalin promoted release of extracellular vesicles and affects mortalin protection from complement-dependent cytotoxicity in breast cancer cells and leukemia cells. Oncotarget 10, 5419-5438. doi: 10.18632/oncotarget. 27138

Huotari, J., and Helenius, A. (2011). Endosome maturation. EMBO J. 30, 34813500. doi: 10.1038/emboj.2011.286

Hurley, J. H. (2015). ESCRTs are everywhere. EMBO J. 34, 2398-2407.

Im, E.-J., Lee, C.-H., Moon, P.-G., Rangaswamy, G. G., Lee, B., Lee, J. M., et al. (2019). Sulfisoxazole inhibits the secretion of small extracellular vesicles by targeting the endothelin receptor A. Nat. Commun. 10, 1387-1387.

Iversen, T. G., Frerker, N., and Sandvig, K. (2012). Uptake of ricinB-quantum dot nanoparticles by a macropinocytosis-like mechanism. J. Nanobiotech. 10, 33-33. doi: 10.1186/1477-3155-10-33

Jorfi, S., Ansa-Addo, E. A., Kholia, S., Stratton, D., Valley, S., Lange, S., et al. (2015). Inhibition of microvesiculation sensitizes prostate cancer cells to chemotherapy and reduces docetaxel dose required to limit tumor growth in vivo. Sci. Rep. 5, 13006-13006.

Kanlikilicer, P., Bayraktar, R., Denizli, M., Rashed, M. H., Ivan, C., Aslan, B., et al. (2018). Exosomal miRNA confers chemo resistance via targeting Cav1/pgp/M2-type macrophage axis in ovarian cancer. EBioMedicine 38, 100-112. doi: 10.1016/j.ebiom.2018.11.004

Khan, F. M., Saleh, E., Alawadhi, H., Harati, R., Zimmermann, W.-H., and El-Awady, R. (2018). Inhibition of exosome release by ketotifen enhances sensitivity of cancer cells to doxorubicin. Cancer Biol. Ther. 19, 25-33. doi: 10.1080/15384047.2017.1394544

Khan, S., Jutzy, J., Aspe, J., Mcgregor, D., Neidigh, J., and Wall, N. (2011). Survivin is released from cancer cells via exosomes. Int. J. Progr. Cell Death 16, 1-12. doi: $10.1007 /$ s10495-010-0534-4 
Kim, H. J., Park, M. K., Kim, S. Y., and Lee, C. H. (2014). Novel Suppressive Effects of Ketotifen on Migration and Invasion of MDA-MB-231 and HT-1080 Cancer Cells. Biomole. Ther. 22, 540-546. doi: 10.4062/biomolther.2014.081

Kirchhausen, T., Macia, E., and Pelish, H. E. (2008). Use of dynasore, the small molecule inhibitor of dynamin, in the regulation of endocytosis. Methods Enzymol. 438, 77-93. doi: 10.1016/s0076-6879(07)38006-3

Koch, R., Aung, T., Vogel, D., Chapuy, B., Wenzel, D., Becker, S., et al. (2016). Nuclear Trapping through Inhibition of Exosomal Export by Indomethacin Increases Cytostatic Efficacy of Doxorubicin and Pixantrone. Clin. Cancer Res. 22:395. doi: 10.1158/1078-0432.ccr-15-0577

Koivusalo, M., Welch, C., Hayashi, H., Scott, C. C., Kim, M., Alexander, T., et al. (2010). Amiloride inhibits macropinocytosis by lowering submembranous $\mathrm{pH}$ and preventing Racl and Cdc42 signaling. J. Cell Biol. 189, 385-385. doi: 10.1083/jcb.20090808620100331c

Kosaka, N., Iguchi, H., Hagiwara, K., Yoshioka, Y., Takeshita, F., and Ochiya, T. (2013). Neutral sphingomyelinase 2 (nSMase2)-dependent exosomal transfer of angiogenic microRNAs regulate cancer cell metastasis. J. Biol. Chem. 288, 10849-10859. doi: 10.1074/jbc.m112.446831

Kosaka, N., Iguchi, H., Yoshioka, Y., Takeshita, F., Matsuki, Y., and Ochiya, T. (2010). Secretory mechanisms and intercellular transfer of microRNAs in living cells. J. Biol. Chem. 285, 17442-17452. doi: 10.1074/jbc.m110.107821

Kosgodage, U. S., Mould, R., Henley, A. B., Nunn, A. V., Guy, G. W., Thomas, E. L., et al. (2018). Cannabidiol (CBD) Is a Novel Inhibitor for Exosome and Microvesicle (EMV) Release in Cancer. Front. Pharmacol. 9:889-889. doi: 10. 3389/fphar.2018.00889

Kosgodage, U. S., Trindade, R. P., Thompson, P. R., Inal, J. M., and Lange, S. (2017). Chloramidine/Bisindolylmaleimide-I-Mediated Inhibition of Exosome and Microvesicle Release and Enhanced Efficacy of Cancer Chemotherapy. Int. J. Mole. Sci. 18:1007. doi: 10.3390/ijms18051007

Kulshreshtha, A., Singh, S., Ahmad, M., Khanna, K., Ahmad, T., Agrawal, A., et al. (2019). Simvastatin mediates inhibition of exosome synthesis, localization and secretion via multicomponent interventions. Sci. Rep. 9:16373.

Lenassi, M., Cagney, G., Liao, M., Vaupotic, T., Bartholomeeusen, K., Cheng, Y., et al. (2010). HIV Nef is secreted in exosomes and triggers apoptosis in bystander CD4+ T cells. Traffic 11, 110-122. doi: 10.1111/j.1600-0854.2009. 01006.x

Li, B., Antonyak, M. A., Zhang, J., and Cerione, R. A. (2012). RhoA triggers a specific signaling pathway that generates transforming microvesicles in cancer cells. Oncogene 31, 4740-4749. doi: 10.1038/onc.2011.636

Lin, H.-P., Singla, B., Ghoshal, P., Faulkner, J. L., Cherian-Shaw, M., O'connor, P. M., et al. (2018). Identification of novel macropinocytosis inhibitors using a rational screen of Food and Drug Administration-approved drugs. Br. J. Pharmacol. 175, 3640-3655. doi: 10.1111/bph.14429

Liu, W., Chen, G., Sun, L., Zhang, Y., Han, J., Dai, Y., et al. (2019). TUFT1 Promotes Triple Negative Breast Cancer Metastasis, Stemness, and Chemoresistance by Up-Regulating the Rac1/ $\beta$-Catenin Pathway. Front. Oncol. 9:617-617. doi: 10. 3389/fonc. 2019.00617

Liu, Y., and Tang, C. (2012). Regulation of ABCA1 functions by signaling pathways. Biochim. Biophys. Acta 1821, 522-529. doi: 10.1016/j.bbalip.2011.08.015

Maacha, S., Bhat, A., Jimenez, L., Raza, A., Haris, M., Uddin, S., et al. (2019). Extracellular vesicles-mediated intercellular communication: roles in the tumor microenvironment and anti-cancer drug resistance. Mole. Cancer 18:55.

Maas, S. L. N., Breakefield, X. O., and Weaver, A. M. (2017). Extracellular Vesicles: Unique Intercellular Delivery Vehicles. Trends Cell Biol. 27, 172-188. doi: 10.1016/j.tcb.2016.11.003

Marleau, A. M., Chen, C.-S., Joyce, J. A., and Tullis, R. H. (2012). Exosome removal as a therapeutic adjuvant in cancer. J. Translat. Med. 10, 134-134.

Martin, K. R., Kantari-Mimoun, C., Yin, M., Pederzoli-Ribeil, M., Angelot-Delettre, F., Ceroi, A., et al. (2016). Proteinase 3 Is a Phosphatidylserine-binding Protein That Affects the Production and Function of Microvesicles. J. Biol. Chem. 291, 10476-10489. doi: 10.1074/jbc.m115.698639

Menck, K., Sönmezer, C., Worst, T. S., Schulz, M., Dihazi, G. H., Streit, F., et al. (2017). Neutral sphingomyelinases control extracellular vesicles budding from the plasma membrane. J. Extrac. Vesicles 6, 1378056-1378056. doi: 10.1080/ 20013078.2017.1378056

Mettlen, M., Chen, P.-H., Srinivasan, S., Danuser, G., and Schmid, S. L. (2018). Regulation of Clathrin-Mediated Endocytosis. Ann. Rev. Biochem. 87, 871-896.
Mineo, M., Garfield, S. H., Taverna, S., Flugy, A., De Leo, G., Alessandro, R., et al. (2012). Exosomes released by K562 chronic myeloid leukemia cells promote angiogenesis in a Src-dependent fashion. Angiogenesis 15, 33-45. doi: 10.1007/ s10456-011-9241-1

Muralidharan-Chari, V., Clancy, J. W., Sedgwick, A., and D'souza-Schorey, C. (2010). Microvesicles: mediators of extracellular communication during cancer progression. J. Cell Sci. 123, 1603-1611. doi: 10.1242/jcs.064386

Muralidharan-Chari, V., Kohan, H. G., Asimakopoulos, A. G., Sudha, T., Sell, S., Kannan, K., et al. (2016). Microvesicle removal of anticancer drugs contributes to drug resistance in human pancreatic cancer cells. Oncotarget 7, 50365-50379. doi: 10.18632/oncotarget.10395

Nabhan, J. F., Hu, R., Oh, R. S., Cohen, S. N., and Lu, Q. (2012). Formation and release of arrestin domain-containing protein 1-mediated microvesicles (ARMMs) at plasma membrane by recruitment of TSG101 protein. Proc. Natl. Acad. Sci. U S A 109, 4146-4151. doi: 10.1073/pnas.1200448109

Namee, N., and O'driscoll, L. (2018). Extracellular vesicles and anti-cancer drug resistance. BBA Rev. Cancer 1870, 123-136. doi: 10.1016/j.bbcan.2018.07.003

Newton, A. J., Kirchhausen, T., and Murthy, V. N. (2006). Inhibition of dynamin completely blocks compensatory synaptic vesicle endocytosis. Proc. Natl. Acad. Sci. U S A 103, 17955-17960. doi: 10.1073/pnas.0606212103

Nishida-Aoki, N., Tominaga, N., Takeshita, F., Sonoda, H., Yoshioka, Y., and Ochiya, T. (2017). Disruption of Circulating Extracellular Vesicles as a Novel Therapeutic Strategy against Cancer Metastasis. Mole. Ther. 25, 181-191. doi: 10.1016/j.ymthe.2016.10.009

O'neill, C. P., Gilligan, K. E., and Dwyer, R. M. (2019). Role of extracellular vesicles (EVs) in cell stress response and resistance to cancer therapy. Cancers 11:136. doi: $10.3390 /$ cancers 11020136

Pfrieger, F. W., and Vitale, N. (2018). Cholesterol and the journey of extracellular vesicles. J. Lipid Res. 59, 2255-2261.

Phuyal, S., Hessvik, N. P., Skotland, T., Sandvig, K., and Llorente, A. (2014). Regulation of exosome release by glycosphingolipids and flotillins. FEBS J. 281, 2214-2227. doi: $10.1111 /$ febs. 12775

Pilzer, D., and Fishelson, Z. (2005). Mortalin/GRP75 promotes release of membrane vesicles from immune attacked cells and protection from complement-mediated lysis. Int. Immunol. 17, 1239-1248. doi: 10.1093/ intimm/dxh300

Richards, K. E., Zeleniak, A. E., Fishel, M. L., Wu, J., Littlepage, L. E., and Hill, R. (2017). Cancer-associated fibroblast exosomes regulate survival and proliferation of pancreatic cancer cells. Oncogene 36, 1770-1778. doi: 10.1038/ onc. 2016.353

Sandvig, K., Kavaliauskiene, S., and Skotland, T. (2018). Clathrin-independent endocytosis: an increasing degree of complexity. Histochem. Cell Biol. 150, 107-118. doi: 10.1007/s00418-018-1678-5

Santos, J., Lima, N., Sarian, L., Matheu, A., Ribeiro, M., and Derchain, S. (2018). Exosome-mediated breast cancer chemoresistance via miR-155 transfer. Sci. Rep. 8, 829-829.

Schlienger, S., Campbell, S., and Claing, A. (2014). ARF1 regulates the Rho/MLC pathway to control EGF-dependent breast cancer cell invasion. Mole. Biol. Cell 25, 17-29. doi: 10.1091/mbc.e13-06-0335

Sedgwick, A. E., Clancy, J. W., Olivia Balmert, M., and D'souza-Schorey, C. (2015). Extracellular microvesicles and invadopodia mediate non-overlapping modes of tumor cell invasion. Sci. Rep. 5:14748.

Sento, S., Sasabe, E., and Yamamoto, T. (2016). Application of a Persistent Heparin Treatment Inhibits the Malignant Potential of Oral Squamous Carcinoma Cells Induced by Tumor Cell-Derived Exosomes. PLoS One 11:e0148454.doi: 10. 1371/journal.pone.0148454

Sexton, R. E., Mpilla, G., Kim, S., Philip, P. A., and Azmi, A. S. (2019). Ras and exosome signaling. Semin. Cancer Biol. 54, 131-137. doi: 10.1016/j.semcancer. 2019.02.004

Si, W., Shen, J., Zheng, H., and Fan, W. (2019). The role and mechanisms of action of microRNAs in cancer drug resistance. Clin. Epigenetics 11, 25-25.

Song, X., Ding, Y., Liu, G., Yang, X., Zhao, R., Zhang, Y., et al. (2016). Cancer Cell-derived Exosomes Induce Mitogen-activated Protein Kinase-dependent Monocyte Survival by Transport of Functional Receptor Tyrosine Kinases. J. Biol. Chem. 291, 8453-8464. doi: 10.1074/jbc.m116.716316

Stratton, D., Moore, C., Zheng, L., Lange, S., and Inal, J. (2015). Prostate cancer cells stimulated by calcium-mediated activation of protein kinase $\mathrm{C}$ undergo a 
refractory period before re-releasing calcium-bearing microvesicles. Biochem. Biophys. Res. Commun. 460, 511-517. doi: 10.1016/j.bbrc.2015.03.061

Tauro, B. J., Greening, D. W., Mathias, R. A., Mathivanan, S., Ji, H., and Simpson, R. J. (2013). Two distinct populations of exosomes are released from LIM1863 colon carcinoma cell-derived organoids. Mole. Cell. Proteom. 12, 587-598. doi: 10.1074/mcp.m112.021303

Taylor, J., and Bebawy, M. (2019). Proteins Regulating Microvesicle Biogenesis and Multidrug Resistance in Cancer. Proteomics 19:1800165. doi: 10.1002/pmic. 201800165

Tian, T., Zhu, Y.-L., Zhou, Y.-Y., Liang, G.-F., Wang, Y.-Y., Hu, F.-H., et al. (2014). Exosome uptake through clathrin-mediated endocytosis and macropinocytosis and mediating miR-21 delivery. J. Biol. Chem. 289, 22258-22267. doi: 10.1074/jbc.m114. 588046

To, K. K., Poon, D. C., Wei, Y., Wang, F., Lin, G., and Fu, L. (2015a). Pelitinib (EKB569) targets the up-regulation of $A B C B 1$ and $A B C G 2$ induced by hyperthermia to eradicate lung cancer. Br. J. Pharmacol. 172, 4089-4106. doi: 10.1111/bph. 13189

To, K. K., Poon, D. C., Wei, Y., Wang, F., Lin, G., and Fu, L. W. (2015b). Vatalanib sensitizes $\mathrm{ABCB} 1$ and $\mathrm{ABCG} 2$-overexpressing multidrug resistant colon cancer cells to chemotherapy under hypoxia. Biochem. Pharmacol. 97, 27-37. doi: 10.1016/j.bcp.2015.06.034

Tricarico, C., Clancy, J., and D'souza-Schorey, C. (2017). Biology and biogenesis of shed microvesicles. Small GTPases 8, 220-232. doi: 10.1080/21541248.2016. 1215283

Vercauteren, D., Vandenbroucke, R. E., Jones, A. T., Rejman, J., Demeester, J., De Smedt, S. C., et al. (2010). The use of inhibitors to study endocytic pathways of gene carriers: optimization and pitfalls. Mole. Ther. 18, 561-569. doi: 10.1038/ mt.2009.281

Wang, Q., and $\mathrm{Lu}$, Q. (2017). Plasma membrane-derived extracellular microvesicles mediate non-canonical intercellular NOTCH signaling. Nat. Commun. 8, 709-709.
Wang, X. Z., Haiyun, Chen, and Xiaozhuo. (2019). Drug resistance and combating drug resistance in cancer. Cancer Drug Res. 2, 141-160.

Wang, Y., Luo, L., Mörgelin, M., and Thorlacius, H. (2017). Racl regulates sepsisinduced formation of platelet-derived microparticles and thrombin generation. Biochem. Biophys. Res. Commun. 487, 887-891. doi: 10.1016/j.bbrc.2017.04.147

Wilson, K. F., Erickson, J. W., Antonyak, M. A., and Cerione, R. A. (2013). Rho GTPases and their roles in cancer metabolism. Trends Mole. Med. 19, 74-82. doi: 10.1016/j.molmed.2012.10.011

Wu, C.-H., Silvers, C. R., Messing, E. M., and Lee, Y.-F. (2019). Bladder cancer extracellular vesicles drive tumorigenesis by inducing the unfolded protein response in endoplasmic reticulum of nonmalignant cells. J. Biol. Chem. 294, 3207-3218. doi: 10.1074/jbc.ra118.006682

Xu, R., Rai, A., Chen, M., Suwakulsiri, W., Greening, D., and Simpson, R. (2018). Extracellular vesicles in cancer - implications for future improvements in cancer care. Nat. Rev. Clin. Oncol. 15, 617-638. doi: 10.1038/s41571-018-0036-9

Yousafzai, N. A., Wang, H., Wang, Z., Zhu, Y., Zhu, L., Jin, H., et al. (2018). Exosome mediated multidrug resistance in cancer. Am. J. Cancer Res. 8, 2210-2226.

Zaborowski, M. P., Balaj, L., Breakefield, X. O., and Lai, C. P. (2015). Extracellular vesicles: composition, biological relevance, and methods of study. Bioscience 65 , 783-797. doi: 10.1093/biosci/biv084

Conflict of Interest: The authors declare that the research was conducted in the absence of any commercial or financial relationships that could be construed as a potential conflict of interest.

Copyright $\odot 2021$ Hayatudin, Fong, Ming, Goh, Lee and Kifli. This is an open-access article distributed under the terms of the Creative Commons Attribution License (CC BY). The use, distribution or reproduction in other forums is permitted, provided the original author(s) and the copyright owner(s) are credited and that the original publication in this journal is cited, in accordance with accepted academic practice. No use, distribution or reproduction is permitted which does not comply with these terms. 